\title{
A Derivative-Based Parser Generator for Visibly Pushdown Grammars
}

\author{
XIAODONG JIA, The Pennsylvania State University, USA \\ ASHISH KUMAR, The Pennsylvania State University, USA \\ GANG TAN, The Pennsylvania State University, USA
}

In this paper, we present a derivative-based, functional recognizer and parser generator for visibly pushdown grammars. The generated parser accepts ambiguous grammars and produces a parse forest containing all valid parse trees for an input string in linear time. Each parse tree in the forest can then be extracted also in linear time. Besides the parser generator, to allow more flexible forms of the visibly pushdown grammars, we also present a translator that converts a tagged CFG to a visibly pushdown grammar in a sound way, and the parse trees of the tagged CFG are further produced by running the semantic actions embedded in the parse trees of the translated visibly pushdown grammar. The performance of the parser is compared with a popular parsing tool ANTLR and other popular hand-crafted parsers. The correctness of the core parsing algorithm is formally verified in the proof assistant Coq.

\section{CCS Concepts: • Software and its engineering $\rightarrow$ Parsers; Software verification; $\bullet$ Theory of computation} $\rightarrow$ Grammars and context-free languages.

Additional Key Words and Phrases: parser generators, formal verification, derivative-based parsing

\section{ACM Reference Format:}

Xiaodong Jia, Ashish Kumar, and Gang Tan. 2021. A Derivative-Based Parser Generator for Visibly Pushdown Grammars. Proc. ACM Program. Lang. 5, OOPSLA, Article 151 (October 2021), 24 pages. https://doi.org/10. $1145 / 3485528$

\section{INTRODUCTION}

Parsing is a fundamental component in computer systems. Modern parsers used in high-performance settings such as web browsers and network routers need to be efficient, as their performance is critical to the performance of the whole system. Furthermore, high-assurance parsers are becoming increasingly more important for security, in settings such as web applications, where their parsers are directly processing potentially adversarial inputs from the network. In these settings, formally verified parsers are highly desirable.

Most parsing libraries are based on Context-Free Grammars (CFGs) or their variants. Although very flexible, CFGs have limitations in terms of efficiency and formal verification. First, not all CFGs can be converted to deterministic pushdown automata (PDA); the inherent nondeterminism in some CFGs causes the worst-case running time of general CFG-based parsing algorithms to be $O\left(n^{3}\right)$. Moreover, formally verifying general CFG parsing algorithms is a difficult task. To our best knowledge, there is no formally verified CFG parsing algorithm due to its complexity.

Authors' addresses: Xiaodong Jia, The Pennsylvania State University, 201 Old Main, State College, Pennsylvania, USA, 16802; Ashish Kumar, The Pennsylvania State University, 201 Old Main, State College, Pennsylvania, USA, 16802; Gang Tan, The Pennsylvania State University, 201 Old Main, State College, Pennsylvania, USA, 16802.

Permission to make digital or hard copies of all or part of this work for personal or classroom use is granted without fee provided that copies are not made or distributed for profit or commercial advantage and that copies bear this notice and the full citation on the first page. Copyrights for components of this work owned by others than ACM must be honored. Abstracting with credit is permitted. To copy otherwise, or republish, to post on servers or to redistribute to lists, requires prior specific permission and/or a fee. Request permissions from permissions@acm.org.

(c) 2021 Association for Computing Machinery.

2475-1421/2021/10-ART151

https://doi.org/10.1145/3485528

Proc. ACM Program. Lang., Vol. 5, No. OOPSLA, Article 151. Publication date: October 2021. 
To achieve efficient parsing, many parsing frameworks place restrictions on what CFGs can be accepted, at the expense of placing the burden on users to refactor their grammars to satisfy those restrictions. Please see the related-work section for discussion about common kinds of restrictions, leading to parsing frameworks such as LL(k), LR(k) (Deremer 1969), PEGs (Parsing Expression Grammars) (Ford 2004), etc.

This paper explores an alternative angle of building parsers based on Visibly Pushdown Grammars (VPGs) (Alur and Madhusudan 2009). In VPGs, users explicitly partition all terminals into three kinds: plain, call, and return symbols. This partitioning makes the conversion of a VPG to a deterministic PDA always possible, which provides the foundation for efficient algorithms. Compared to requiring users to refactor their grammars to satisfy restrictions placed by parsing frameworks such as $\mathrm{LL}(\mathrm{k})$ asking users to specify what nonterminals are call and return symbols is less of a burden.

VPGs have been used in program analysis, XML processing, and other fields, but their potential in parsing has not been fully exploited. In this paper, we show that VPGs bring many benefits in parsing. First, we show an efficient, linear-time parsing algorithm for VPGs. Second, our algorithm is amenable to formal verification. Overall, this paper makes the following contributions.

- We present a derivative-based algorithm for VPG recognition and parsing. The algorithm is guaranteed to run in linear time. The generated parser accepts ambiguous grammars and produces a parse forest for the input string, where each parse tree in the forest can be extracted in linear time.

- We mechanize the correctness proofs of the parsing algorithm in Coq.

- We present a surface grammar called tagged CFGs to allow more convenient use of our parsing framework. Users can use their familiar CFGs for developing grammars and provide additional tagging information on nonterminals. A sound translator then converts a tagged CFG to a VPG.

The remainder of this paper is organized as follows. We first introduce VPGs in Section 2 and discuss related work in Section 3. Section 4 presents a derivative-based VPG recognizer, which enlightens the parsing algorithm discussed in Section 5. The translator and tagged CFGs are discussed in Section 6. We then evaluate the VPG parser in Section 7.

\section{BACKGROUND}

As a class of grammars, VPGs (Alur and Madhusudan 2009) have been used in program analysis, XML processing, and other fields. Compared with CFGs, VPGs enjoy many good properties. It is always possible to build a deterministic PDA from a VPG. The terminals in a VPG are partitioned into three kinds and the stack action associated with an input symbol is fully determined by the kind of the symbol: an action of pushing to the stack is always performed for a call symbol, an action of popping from the stack is always performed for a return symbol, and no stack action is performed for a plain symbol. Furthermore, VPGs enjoy all closure properties, including intersection and complement. As will be shown in this paper, these properties enable the building of linear-time parsers for VPGs, and make VPGs amenable to formal verification. The expressive power of VPG is between regular grammars and CFGs, and is sufficient for describing the syntax of many practical languages, such as JSON, XML, and HTML.

We next give a formal account of VFGs. A grammar $G$ is represented as a tuple $\left(V, \Sigma, P, L_{0}\right)$, where $V$ is the set of nonterminals, $\Sigma$ is the set of terminals, $P$ is the set of production rules, and $L_{0} \in V$ is the start symbol. The alphabet $\Sigma$ is partitioned into three sets: $\Sigma_{l}, \Sigma_{c}, \Sigma_{r}$, which contain plain, call and return symbols, respectively. Notation-wise, a terminal in $\Sigma_{c}$ is tagged with $\langle$ on the left, and a terminal in $\Sigma_{r}$ is tagged with $\rangle$ on the right. For example, $\left\langle a\right.$ is a call symbol in $\Sigma_{c}$, and $b\rangle$ is a return symbol in $\Sigma_{r}$. 
We first formally define well-matched VPGs. Intuitively, a well-matched VPG generates only well-matched strings, in which a call symbol is always matched with a return symbol in a derived string.

Definition 2.1 (Well-matched VPGs). A grammar $G=\left(V, \Sigma, P, L_{0}\right)$ is a well-matched VPG with respect to the partitioning $\Sigma=\Sigma_{l} \cup \Sigma_{c} \cup \Sigma_{r}$, if every production rule in $P$ is in one of the following forms.

(1) $L \rightarrow \epsilon$, where $\epsilon$ stands for the empty string;

(2) $L \rightarrow c L_{1}$, where $c \in \Sigma_{l}$;

(3) $L \rightarrow\left\langle a L_{1} b\right\rangle L_{2}$, where $\left\langle a \in \Sigma_{c}\right.$ and $\left.b\right\rangle \in \Sigma_{r}$.

Note that in $L \rightarrow c L_{1}$ terminal $c$ must be a plain symbol, and in $L \rightarrow\left\langle a L_{1} b\right\rangle L_{2}$ a call symbol must be matched with a return symbol; these requirements ensure that any derived string must be well-matched.

The following is an example of a well-matched VPG, which is taken from a VPG for XML:

$$
\text { element } \rightarrow \text { OpenTag content CloseTag | SingleTag }
$$

In this example, nonterminals start with a lowercase character, such as "element", and terminals start with an uppercase character, such as "OpenTag". The grammar shows a typical usage of VPGs to model a hierarchically nested matching structure of XML texts: "OpenTag" is matched with "CloseTag", and "content" nested in between can be "element" itself (not shown in the above snippet) and forms an inner hierarchy.

In the rest of the paper, we use the term VPGs for well-matched VPGs and use the term general VPGs to allow the case of pending calls and returns, which means that a call/return symbol may not have its corresponding return/call symbol in the input string. To accommodate pending symbols, general VPGs, in addition, allow rules in the forms of $L \rightarrow\left\langle a L^{\prime}\right.$ and $\left.L \rightarrow b\right\rangle L^{\prime}$, which we call pending rules. Further, the set of nonterminals $V$ is partitioned into $V^{0}$ and $V^{1}$ : nonterminals in $V^{0}$ only generate well-matched strings, while nonterminals in $V^{1}$ can generate strings with pending symbols.

Definition 2.2 (General VPGs). A grammar $G=\left(V, \Sigma, P, L_{0}\right)$ is a general VPG with respect to the partitioning $\Sigma=\Sigma_{l} \cup \Sigma_{c} \cup \Sigma_{r}$ and $V=V^{0} \cup V^{1}$, if every rule in $P$ is in one of the following forms:

(1) $L \rightarrow \epsilon$;

(2) $L \rightarrow i L_{1}$, where $i \in \Sigma$, and if $L \in V^{0}$ then (1) $i \in \Sigma_{l}$ and (2) $L_{1} \in V^{0}$;

(3) $L \rightarrow\left\langle a L_{1} b\right\rangle L_{2}$, where $\left\langle a \in \Sigma_{c}, b\right\rangle \in \Sigma_{r}, L_{1} \in V^{0}$, and if $L \in V^{0}$, then $L_{2} \in V^{0}$.

The above definition imposes constraints on how $V^{0}$ and $V^{1}$ nonterminals can be used in a rule. For example, in $L \rightarrow\left\langle a L_{1} b\right\rangle L_{2}$, nonterminal $L_{1}$ must be a well-matched nonterminal; so $P$ cannot include rules such as $L_{1} \rightarrow\left\langle a L_{3}\right.$, since $L_{1}$ is supposed to generate only well-matched strings.

The notion of a derivation in VPGs is the same as the one in CFGs. We write $w \rightarrow w^{\prime}$ to mean a single derivation step according to a grammar, where $w$ and $w^{\prime}$ are strings of terminals or nonterminals. We write $L \rightarrow^{*} w$ to mean that $w$ can be derived from $L$ via a sequence of derivation steps.

\section{RELATED WORK}

Most parser libraries rely on the formalism of Context-Free Grammars (CFGs) and user-defined semantic actions for generating parse trees. Many CFG-based parsing algorithms have been proposed in the past, including LL(k), LR(k) (Deremer 1969), Earley (Earley 1970), CYK (Cocke 1969; Kasami 1965; Younger 1967), among many others. LL(k) and LR(k) algorithms are commonly used, but their input grammars must be unambiguous. Users often have to change/refactor their grammars to 
avoid conflicts in $\mathrm{LL}(\mathrm{k})$ and $\mathrm{LR}(\mathrm{K})$ parsing tables, a nontrivial task. Earley, CYK, and GLR parsing can handle any CFG, but their worst-case running time is $O\left(n^{3}\right)$. In contrast, our VPG parsing accepts ambiguous grammars and is linear time.

Our VPG parsing algorithm relies on derivatives. One major benefit of working with derivatives is that it is amenable to formal verification, as proofs related to derivatives involve algebraic transformations on symbolic expressions; they are easier to develop in proof assistants than it is to reason about graphs (required when formalizing LL and LR algorithms). Brzozowski (1964) first presented the concept of derivatives and used it to build a recognizer for regular expressions. The idea was revived by Owens et al. (2009) and generalized to generate parsers for CFGs (Might et al. 2011), with an exponential worst-case time complexity. More recently, a symbolic approach (Henriksen et al. 2019) for parsing CFGs with derivatives was presented, with cubic time complexity. Finally, Edelmann et al. (2020) presented a formally verified, derivative-based, linear-time parsing algorithm for LL(1) context-free expressions.

Owl is an open-source project ${ }^{1}$ that provides a parser generator for VPGs. It has the same goal as our work, but differs in the following critical aspects: (1) Owl supports only well-matched VPGs, while our parsing library supports full VPGs; (2) Owl adopts a different algorithm and is implemented in an imperative way, while our parsing library is derivative-based and functional; (3) Owl does not provide formal assurance, while our parsing library is formally verified in Coq; (4) Owl rejects ambiguous VPGs, while our parsing library accepts ambiguous grammars and generates parse forests; and (5) Owl does not support semantic actions embedded in grammars, while our parsing library accepts semantic actions.

Due to parsers' importance to security, many efforts have been made to build secure and correct parsers. One obvious approach is testing, through fuzz testing or differential testing (e.g., Petsios et al. (2017)). However, testing cannot show the absence of bugs. Formal verification has also been applied to the building of high-assurance parsers. Jourdan et al. (2012) applied the methodology of translation validation and implemented a verified parser validator for LR(1) grammars. RockSalt (Morrisett et al 2012) included a verified parser for regular expression based DSL. Lasser et al. (2019) and Edelmann et al. (2020) presented verified LL(1) parsers but we are not aware of fully verified LL(k) parsers. Lasser et al. (2021) implemented a verified ALL $\left(^{*}\right)$ parser, which is the algorithm behind ANTLR4 Koprowski and Binsztok (2011) implemented a formally verified parser generator for Parsing Expression Grammars (PEG). Ramananandro et al. (2019) presented a verified parser generator for tag-length-value binary message format descriptions. We formalize our derivative-based, VPG parsing algorithm and its correctness proofs in Coq.

\section{VPG BASED RECOGNITION}

We next present an algorithm for converting a VPG into a deterministic PDA using a derivativebased algorithm. The resulting PDA accepts the same set of strings as the input VPG.

Before we present the formal conversion process, we discuss informally the intuition of the states, the stack, and the transition function of the PDA that is converted from an input VPG. A state in the resulting PDA is a subset of $V \times V$, i.e., a set of nonterminal pairs. A nonterminal pair $\left(L_{1}, L_{2}\right)$ tells that the next part of the input should match $L_{2}$ and the current context is $L_{1}$. The context is the nonterminal that is used to derive $L_{2}$, without consuming an unmatched call symbol before getting to $L_{2}$. Formally, it means there exists a derivation sequence $L_{1} \rightarrow^{*} \omega_{1} L_{2} \omega_{2}$, where $\omega_{1}$ is a sequence of terminals and does not contain an unmatched call symbol, and $\omega_{2}$ is a sequence of terminals or nonterminals.

$\overline{{ }^{1} \text { https://github.com/ianh/owl }}$ 
To give an example, suppose we have the following VPG rules, with $L_{0}$ being the start symbol. We omit the rules for $L_{2}$ and $L_{3}$, which are irrelevant for the discussion.

$$
L_{0} \rightarrow c L_{1} ; L_{1} \rightarrow\left\langle a L_{2} b\right\rangle L_{3} .
$$

The start state of the PDA should be $\left\{\left(L_{0}, L_{0}\right)\right\}$, meaning that the input string should match $L_{0}$ and the context is also $L_{0}$ since $L_{0}$ can be derived from itself (in zero steps) without generating an unmatched call symbol. Given that start state, if the next input symbol is $c$, then the PDA should transition to state $\left\{\left(L_{0}, L_{1}\right)\right\}$; that is, the rest of the input should match $L_{1}$ and the context is still $L_{0}$, since $L_{1}$ is derived from $L_{0}$ without generating an unmatched call symbol. Now suppose the next input char is $\left\langle a\right.$; then the next state should be $\left\{\left(L_{2}, L_{2}\right)\right\}$; notice that there is a context switch as there is an unmatched call symbol that is encountered when going from $L_{1}$ to $L_{2}$ using the rule $L_{1} \rightarrow\left\langle a L_{2} b\right\rangle L_{3}$. As we will show, for this transition, the PDA will also push $\left\{\left(L_{0}, L_{1}\right)\right\}$ and $\langle a$ to the stack, so that when the return symbol $b\rangle$ is encountered, we can use that stack information to look up the old context and transition the PDA to state $\left\{\left(L_{0}, L_{3}\right)\right\}$.

For this example, all states contain just one pair. In general, a state may contain multiple pairs because of possible ambiguity. For example, imagine there is an additional rule $L_{0} \rightarrow c L_{4}$; then from start state $\left\{\left(L_{0}, L_{0}\right)\right\}$, after encountering $c$, the PDA transitions to state $\left\{\left(L_{0}, L_{3}\right),\left(L_{0}, L_{4}\right)\right\}$, reflecting that the rest of the input can match either $L_{3}$ or $L_{4}$.

Given the above discussion, we have the following PDA states and stacks.

Definition 4.1 (PDA states and stacks). Given a VPG, we introduce a PDA whose states are subsets of $V \times V$ and whose stack contains stack symbols of the form $[S,\langle a]$, where $S$ is a PDA state and $\left\langle a \in \Sigma_{c}\right.$ a call symbol. We write $\perp$ for the empty stack, and $[S,\langle a] \cdot T$ for a stack whose top is $[S,\langle a]$ and the rest is $T$. Intuitively, the stack remembers a series of past contexts, which are used for matching future return symbols. We call a pair $(S, T)$ a configuration, with $S$ being the state and $T$ being the stack.

We next utilize the notion of derivatives (Brzozowski 1964; Might et al. 2011; Owens et al. 2009) to compute a recognizer PDA that accepts the same language (i.e., a set of strings) as an input VPG. The derivative of a language $\mathcal{L}$ with respect to an input symbol $c$ is the residual set of strings of those in $\mathcal{L}$ that start with $c$ :

$$
\delta_{c}(\mathcal{L})=\{w \mid c w \in \mathcal{L}\}
$$

As we will show in Definition 4.3, a recognizer PDA configuration $(S, T)$ stands for a language. Transferring the general definition of derivatives to recognizer PDA configurations produces a set of derivative functions, discussed next.

Given a VPG $G=\left(V, \Sigma, P, L_{0}\right)$, we define three kinds of derivative functions: (1) $\delta_{c}$ is for when the next input symbol is a plain symbol $c$; (2) $\delta_{\langle a}$ for when the next input symbol is a call symbol $\langle a$; and (3) $\delta_{b\rangle}$ for when the next input symbol is a return symbol $\left.b\right\rangle$. Each function takes the current state $S$ and the top stack symbol, and returns a new state as well as an action on the stack (expressed as a lambda function). Note that $\delta_{c}$ and $\delta_{\langle a}$ do not need information from the stack; therefore we omit the top stack symbol from their parameters.

Definition 4.2 (Derivative functions).

(1) $\delta_{c}(S)=\left(S^{\prime}, \lambda T . T\right)$, where

$$
S^{\prime}=\left\{\left(L_{1}, L_{3}\right) \mid \exists L_{2},\left(L_{1}, L_{2}\right) \in S \wedge\left(L_{2} \rightarrow c L_{3}\right) \in P\right\} ;
$$

For a plain symbol $c \in \Sigma_{l}$, it checks each pair $\left(L_{1}, L_{2}\right)$ in the current state $S$, and if there is a rule $L_{2} \rightarrow c L_{3}$, pair $\left(L_{1}, L_{3}\right)$ becomes part of the new state. In addition, the stack is left unchanged. 


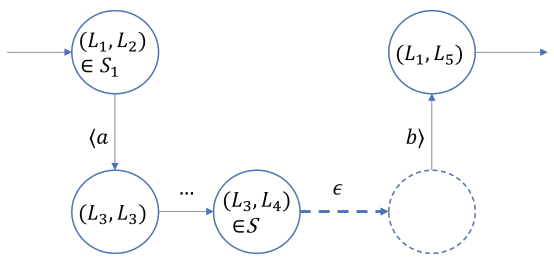

Fig. 1. An example of state transitions: $\left(L_{1}, L_{2}\right)$ transfers to $\left(L_{3}, L_{3}\right)$ with symbol $\langle a$ and there is a rule $L_{2} \rightarrow\left\langle a L_{3} b\right\rangle L_{5}$, and finally transfers to $\left(L_{1}, L_{5}\right)$ with symbol $\left.b\right\rangle$.

(2) $\delta_{\langle a}(S)=\left(S^{\prime}, \lambda T \cdot[S,\langle a] \cdot T)\right.$, where

$$
S^{\prime}=\left\{\left(L_{3}, L_{3}\right) \mid \exists L_{1} L_{2},\left(L_{1}, L_{2}\right) \in S \wedge \exists L_{4},\left(L_{2} \rightarrow\left\langle a L_{3} b\right\rangle L_{4}\right) \in P\right\}
$$

For a call symbol $\left\langle a \in \Sigma_{c}\right.$, it checks each pair $\left(L_{1}, L_{2}\right)$ in the current state $S$; if there is a rule $L_{2} \rightarrow\left\langle a L_{3} b\right\rangle L_{4}$, pair $\left(L_{3}, L_{3}\right)$ becomes part of the new state; note there is a context change since a call symbol is encountered. In addition, the old state together with $\langle a$ is pushed to the stack.

(3) $\delta_{b\rangle}\left(S,\left[S_{1},\langle a]\right)=\left(S^{\prime}\right.\right.$, tail $)$, where

$$
S^{\prime}=\left\{\left(L_{1}, L_{5}\right) \mid \exists L_{2} L_{3} L_{4},\left(L_{1}, L_{2}\right) \in S_{1} \wedge\left(L_{3}, L_{4}\right) \in S \wedge\left(L_{4} \rightarrow \epsilon\right) \in P \wedge\left(L_{2} \rightarrow\left\langle a L_{3} b\right\rangle L_{5}\right) \in P\right\} .
$$

For a return symbol $b\rangle \in \Sigma_{r}$ and a stack top $\left[S_{1},\langle a]\right.$, it checks each pair $\left(L_{1}, L_{2}\right)$ in the state $S_{1}$ of the stack top symbol; if there is a pair $\left(L_{3}, L_{4}\right)$ in the current state $S, L_{4}$ can derive the empty string, and there is a rule $L_{2} \rightarrow\left\langle a L_{3} b\right\rangle L_{5}$, then pair $\left(L_{1}, L_{5}\right)$ becomes part of the new state; note that it checks $L_{4} \rightarrow \epsilon$ to ensure that the current level is finished before returning to the upper level. In addition, the stack top is popped from the stack. Figure 1 presents a drawing that depicts the situation when a return symbol is encountered.

We formalize the semantics of PDA configurations as sets of accepted strings:

Definition 4.3 (Semantics of PDA configurations). We write $(S, T) \sim w$ to mean that a terminal string $w$ can be accepted by the configuration $(S, T)$. It is defined as follows.

(1) $(S, \perp) \leadsto w$ if $\exists\left(L_{1}, L_{2}\right) \in S$, s.t. $L_{2} \rightarrow^{*} w$,

(2) $\left(S,\left[S^{\prime},\langle a] \cdot T^{\prime}\right) \leadsto w_{1} b\right\rangle w_{2}$ if $\exists\left(L_{3}, L_{4}\right) \in S$ s.t.

(a) $L_{4} \rightarrow^{*} w_{1}$ and

(b) $\exists\left(L_{1}, L_{2}\right) \in S^{\prime}, \exists L_{5},\left(L_{2} \rightarrow\left\langle a L_{3} b\right\rangle L_{5}\right) \in P \wedge\left(\left\{\left(L_{1}, L_{5}\right)\right\}, T^{\prime}\right) \leadsto w_{2}$.

The correctness of derivative functions is stated in the following theorem, whose correctness proof is detailed in Appendix A of the companion technical report (Jia et al. 2021). Take the case of $\delta_{c}$ as an example: the theorem states that $(S, T)$ matches $c w$ iff the configuration after running $\delta_{c}$ matches $w$ (the string after consuming $c$ ).

\section{Theorem 4.1 (Derivative Function CORRectness).}

- Assume $\delta_{c}(S)=\left(S^{\prime}, \lambda T . T\right)$ for a plain symbol c. Then $(S, T) \leadsto c w$ iff $\left(S^{\prime}, T\right) \sim w$.

- Assume $\delta_{\langle a}(S)=\left(S^{\prime}, \lambda T\right.$. $[S,\langle a] \cdot T)$ for a call symbol $\left\langle a\right.$. Then $(S, T) \leadsto\left\langle\right.$ aw iff $\left(S^{\prime},[S,\langle a] \cdot T) \leadsto\right.$ w.

- Assume $\delta_{b\rangle}\left(S,\left[S_{1},\langle a]\right)=\left(S^{\prime}\right.\right.$, tail $)$ for a return symbol $\left.b\right\rangle$. Then $\left(S,\left[S_{1},\langle a] \cdot T\right) \leadsto b\right\rangle w$ iff $\left(S^{\prime}, T\right) \leadsto w$. 


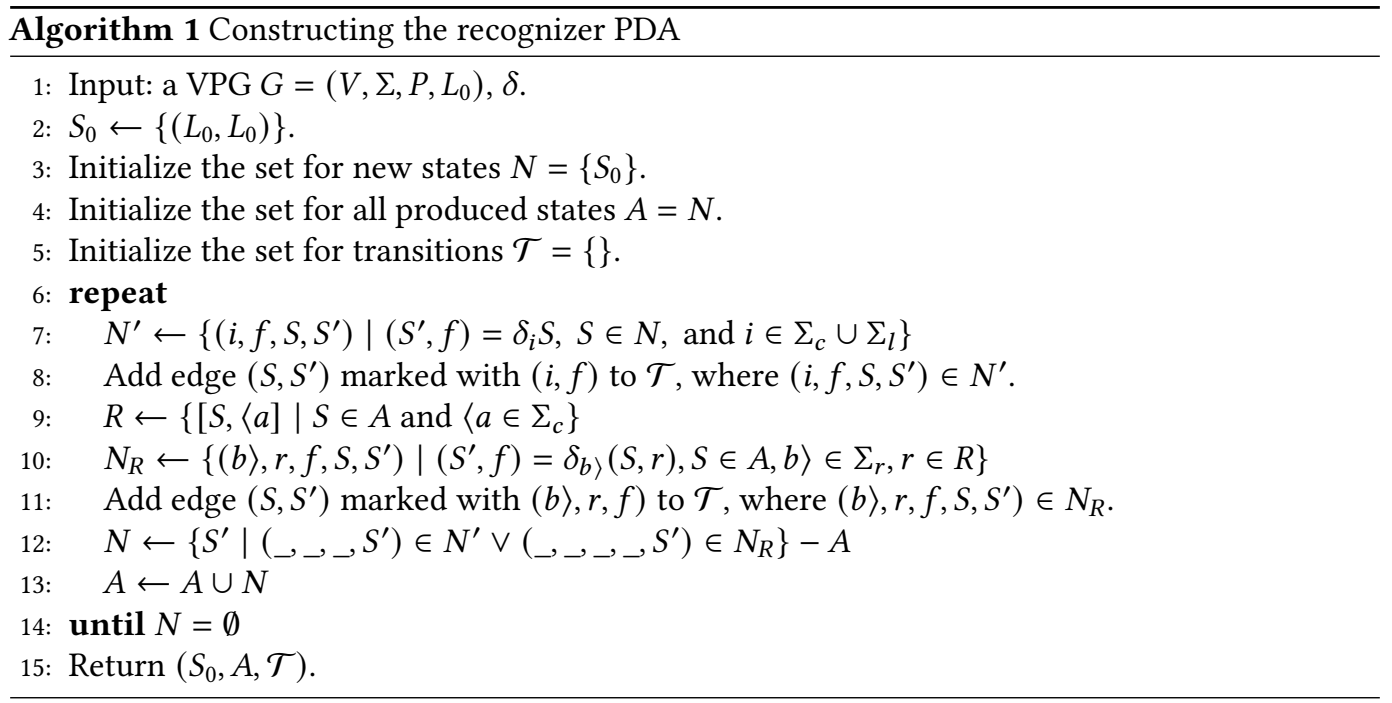

$$
L \rightarrow\langle a A b\rangle L|\epsilon ; A \rightarrow c C| c D ; C \rightarrow c E ; D \rightarrow d E ; E \rightarrow \epsilon
$$

Fig. 2. An example VPG.

With those derivative functions, we can convert a VPA to a PDA, whose set of states is the least solution of the following equation; it makes sure that states are closed under derivatives.

$$
\begin{aligned}
A= & A \cup\left\{S^{\prime} \mid c \in \Sigma_{l}, S \in A, \delta_{c}(S)=\left(S^{\prime}, f\right)\right\} \\
& \cup\left\{S^{\prime} \mid\left\langle a \in \Sigma_{c}, S \in A, \delta_{\langle a}(S)=\left(S^{\prime}, f\right)\right\}\right. \\
& \cup\left\{S^{\prime}|b\rangle \in \Sigma_{r},\left\langle a \in \Sigma_{c}, S \in A, S^{\prime} \in A, \delta_{b\rangle}\left(S,\left[S^{\prime},\langle a]\right)=\left(S^{\prime}, f\right)\right\}\right.\right.
\end{aligned}
$$

We note that the least solution to the previous equation may include unreachable states, since the last line of the equation considers all $\left(S,\left[S^{\prime},\langle a]\right)\right.$ without regard for whether such a configuration is possible. This may make the resulting PDA contain more states and occupy more space for the PDA representation than necessary. However, unreachable states do not affect the linear-time parsing guarantee of VPG parsing, as during parsing those unreachable states are not traversed; further, during experiments we did not experience space issues when representing PDA states and transitions.

Algorithm 1 is an iteration-based method to solve the equation for the least solution, where the returned $S_{0}$ is the initial state, $A$ is the set of all states, and $\mathcal{T}$ is the set of edges between states. For an iteration, $N$ is the set of states that the algorithm should perform derivatives on. Line 7 then performs derivatives using call and plain symbols and line 10 performs derivatives using return symbols.

At the end of each iteration, the following invariants are maintained: (1) $N \subseteq A$; (2) for state $S \in A-N$ and $i \in \Sigma_{c} \cup \Sigma_{l}$, if $\delta_{i}(S)=\left(S^{\prime}, f\right)$, then $S^{\prime} \in A$; (3) for states $S, S^{\prime} \in A-N,\left\langle a \in \Sigma_{c}\right.$, and $b\rangle \in \Sigma_{r}$, if $\delta_{b\rangle}\left(S,\left[S^{\prime},\langle a]\right)=\left(S^{\prime \prime}, f\right)\right.$, then $S^{\prime \prime} \in A$. With these invariants, when $N$ becomes empty, $A$ is closed under derivatives.

As an example, consider the VPG in Figure 2. The PDA generated by Algorithm 1 for this VPG is shown in Figure 3. The input symbols and stack actions are marked on the edges. 


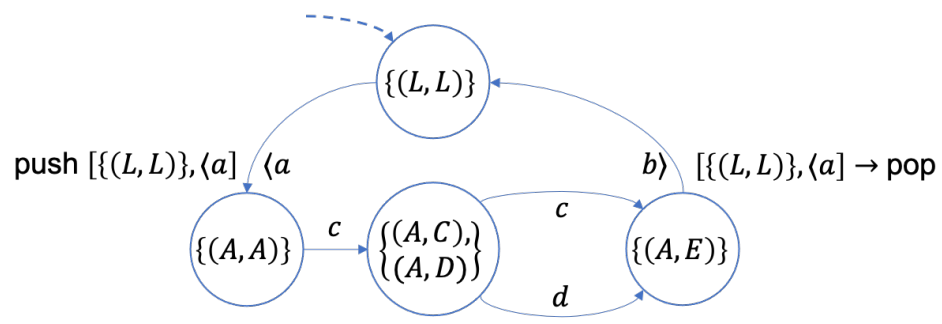

Fig. 3. The PDA generated by Algorithm 1 for grammar in Figure 2. "push $[\{(L, L),\langle a\}]$ " means pushing $[\{(L, L),\langle a\}]$ to the stack, and " $[\{(L, L),\langle a\}] \rightarrow$ pop" means removing the top of the stack, when it is $[\{(L, L),\langle a\}]$.

Once the PDA is constructed from a VPG, it can be run on an input string in a standard way. Then PDA correctness can be stated as follows. We detail PDA execution and the correctness proof in Appendix A of the companion technical report (Jia et al. 2021).

Theorem 4.2 (PDA CORRectness). For VPG $G$ and its start symbol $L_{0}$, a string $w \in \Sigma^{*}$ can be derived from $L_{0}$ (i.e. $L_{0} \rightarrow^{*} w$ ) iff $w$ is accepted by the corresponding PDA.

For converting general VPGs (i.e., with pending rules) to PDA, a couple of changes need to be made on the derivative-based approach: (1) the derivative functions need to consider also pending rules; (2) the acceptance stack may be nonempty because of pending call symbols. The construction is discussed in Appendix B of the companion technical report (Jia et al. 2021).

\section{VPG BASED PARSING}

Parsing is a process to build the parse trees of a given string based on an input grammar. It is equivalent to finding the sequences of rules that can generate the input string. Our VPG-based parsing framework is largely enlightened by the recognizer construction in Section 4. Observe that the execution of the recognizer PDA on an input string $w$ can be represented as a trace of runtime configurations: $\left(S_{0}, T_{0}\right) \stackrel{w_{1}}{\longrightarrow}\left(S_{1}, T_{1}\right) \stackrel{w_{2}}{\longrightarrow}\left(S_{2}, T_{2}\right) \stackrel{w_{3}}{\longrightarrow} \cdots$, where $\left(S_{i}, T_{i}\right)$ is the configuration after consuming $w_{i}$, the $i^{\text {th }}$ symbol of $w$. Each transition in the trace is because of a set of possible rules in the input VPG; therefore, we can augment the configuration trace with information about what rules can be applied during each transition, which gives $\left(S_{0}, T_{0}\right) \stackrel{w_{1}}{\longrightarrow}\left(\left(S_{1}, T_{1}\right), m_{1}\right) \stackrel{w_{2}}{\longrightarrow}\left(\left(S_{2}, T_{2}\right), m_{2}\right) \stackrel{w_{3}}{\longrightarrow}$ $\cdots$, where $m_{i}$ is the set of possible rules at step $i$. With the augmented configuration trace, we can construct parse trees for the input string.

Given this intuition, we could build a parser directly based on the recognizer. However, to reduce the burden of formally verifying the parser, we make several trade-offs in designing the parsing algorithm: (1) instead of extending the recognizer, we present a way to construct the parser PDA independently; as will be shown in Section 5.4, this allows us to formalize correctness in a natural way; (2) we replace the context nonterminal in the recognizer state with a boolean value when constructing the parser; this simplifies formal verification, but may introduce invalid edges in the parse forest; as a result, our design adds a pruning step to prune invalid edges.

\subsection{Overview of VPG-Based Parsing}

At a high level, our VPG-based parsing framework takes an input VPG and generates three components: (1) a parser PDA, which takes an input string and constructs a parse forest representing possibles parses of the string according to the VPG; (2) a pruner PDA, which takes the parse forest 


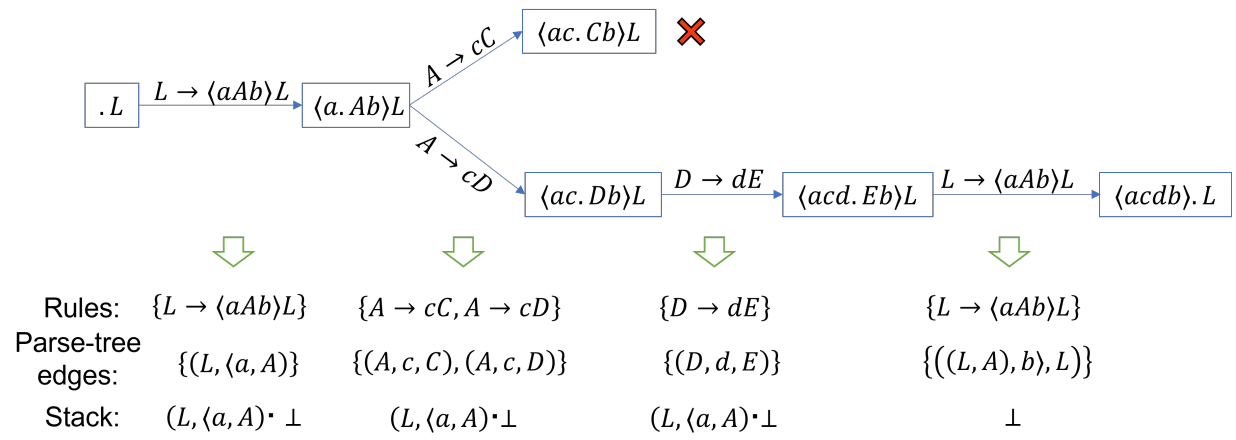

Fig. 4. The parsing process for string $\langle a c d b\rangle$ for the grammar in Figure 2. There are two traces. The first one, followed by a red cross mark, becomes invalid after step 2. Possible rules applied in each step are collected in a set, which is converted to the set of parse-tree edges at that step. The stack keeps track of previous rules that generated call symbols, so that later they can be applied to generate the corresponding return symbols.

and removes invalid edges; (3) an extractor, which takes the pruned parse forest and extracts parse trees.

Before discussing these steps in detail, we use the well-matched VPG in Figure 2 to illustrate the steps of the parser and the pruner PDAs. Let $w=\langle a c d b\rangle$ be the string to parse. Figure 4 visualizes the high-level steps of how our VPG parser parses $w$. Nodes in the figure are sentential forms with a dot indicating the parsing position. The prefix before the dot in a sentential form is the input seen so far; the nonterminal immediately after the dot is the one to parse next; the remainder of the sentential form is actually represented by the stack in our parser, but for ease of understanding we also add it to the nodes in Figure 4. The figure also shows for each step the set of possible rules, those rules represented as parse-tree edges, and the stack after the step.

As an example, after symbol $\langle a$, we have the sentential form $\langle a . A b\rangle L$, where $\langle a$ is the already parsed input, $A$ is the nonterminal to parse, and $b\rangle L$ is the remainder. For this step, only one rule is possible: $L \rightarrow\langle a A b\rangle L$. To represent such a rule used in building a parse tree, we use a triple instead of the rule directly; the reason is to differentiate when $\langle a$ is generated by a matching rule from when $b\rangle$ is generate by the same rule. For example, the parsing of $\langle a$ using $L \rightarrow\langle a A b\rangle L$ is converted to triple $(L,\langle a, A)$. And the remainder is represented by a stack element $(L,\langle a, A)$, which tells us that a rule such as $L \rightarrow\langle a A b\rangle L$ was used to create this stack element; with this information, at step 4 of Figure 4, the parser knows that the next symbol should match $b\rangle$ and, after that, $L$. So the rule $L \rightarrow\langle a A b\rangle L$ is used twice: at step 1 for $\langle a$ and step 4 for $b\rangle$.

As another example, for the second symbol "c" in the input string, there are two possible rules. One with $C$ as the next nonterminal to parse, and the other with $D$. However, notice here in both cases the remainders are $b\rangle L$ and represented by the same stack. This example shows the crucial difference between VPG parsing and CFG parsing. In a general CFG parsing algorithm such as GLR, each possibility has its own stack, reflecting the nondeterministic nature of when stacks are changed in CFGs. In contrast, in VPG parsing all possibilities in one step share the same stack, enabled by the VPG property that the stack is changed when consuming only call/return symbols; as a result, the stack can be shared and factored out.

From this example, we can see that, given an input string $w$ of length $n$, the parser PDA generates a set of possible rules for the $i$-th input symbol; since each rule is represented by a triple in our parser, each step generates a set of triples $m_{i}$. We call the sequence of $\left[m_{1}, \ldots, m_{n}\right]$ the parse forest for $w$; from the sequence, we can recover all parse trees for $w$. We call $\left[e_{1}, \ldots, e_{k}\right]$, where 
$e_{i} \in m_{i}$ for $i \in[1 . . k]$, a trace of parse-tree edges; when $k=n$, it is a complete trace. A complete trace is a linear representation of a candidate parse tree. For example, a complete trace in Figure 4 is $[(L,\langle a, A),(A, c, D),(D, d, E),((L, A), b\rangle, L)]$, representing a candidate parse tree. Although the number of traces may grow exponentially with the length of the input string ${ }^{2}$, the set of distinct rules possible at each step is always finite and bounded by the size of the input grammar. As a result, the parse forest representation is linear to the size of the input string.

Notice that the first trace in Figure 4 is followed by a red cross mark, because the sentential form $\langle a c . C b\rangle L$ cannot be followed by any rule to generate $d$. However, the parse forest still keeps the invalid edge $(A, c, C)$. In our approach, we use a pruner PDA to prune invalid parsetree edges in the parse forest. For example, the pruned parse forest for the above example is $[\{(L,\langle a, A)\},\{(A, c, D)\},\{(D, d, E)\},\{((L, A), b\rangle, E)\}]$.

Among the three components of the VPG parsing framework, the first two can fail: the parser PDA may fail because it cannot make a transition with the next input symbol; the pruner PDA may prune the parse forest to an empty parse forest, meaning that the input string cannot be parsed.

\subsection{The Parser PDA}

As discussed earlier, a parse tree for a VPG can be represented by a linear sequence of triples, each representing an edge in the parse tree. E.g., when rule $L \rightarrow\left\langle a L_{1}\right.$ is used, it is represented as $\left(L,\left\langle a, L_{1}\right)\right.$. However, for general VPGs with pending rules, such triples are insufficient. For example, ( $L,\left\langle a, L_{1}\right)$ can be the result from the rule $L \rightarrow\left\langle a L_{1}\right.$, or the rule $L \rightarrow\left\langle a L_{1} b\right\rangle L_{2}$. We need to further differentiate pending rule edges and matching rule edges, since pending rules cannot be used within matching rules, required by general VPGs (Definition 2.2).

Our solution is to tag every nonterminal in a parse-tree edge a boolean $u$; a similar notion called linear acceptance is discussed by Alur and Madhusudan (2009). Let $L$ be a nonterminal; a tagged $L$ is written as $L^{u}$. Intuitively, $L^{\text {true }}$ generates only well-matched strings and can use only well-matched rules when generating call/return symbols; in contrast, $L^{\text {false }}$ can also use pending rules when generating call/return symbols. For a general VPG, if the parser uses a well-matched rule $L \rightarrow\left\langle a L_{1} b\right\rangle L_{2}$ to match $\left\langle a\right.$, then it has to use $L_{1}^{\text {true }}$ to perform parsing next, since general VPGs require that $L_{1}$ must generate well-matched strings.

With the above discussion, parse-tree edges for general VPGs can be defined as follows:

Definition 5.1 (The edges $\left.\mathcal{M}_{\text {pln }}, \mathcal{M}_{\text {call }}, \mathcal{M}_{\text {ret }}\right)$. Given a VPG $G=\left(\Sigma, V, P, L_{0}\right)$,

(1) the set of plain edges, denoted as $\mathcal{M}_{\mathrm{pln}}$, is defined as $\left\{\left(L^{u}, c, L_{1}^{u}\right) \mid\left(L \rightarrow c L_{1}\right) \in P\right\}$;

(2) the set of call edges, denoted as $\mathcal{M}_{\text {call }}$, is defined as $\left\{\left(L^{u},\left\langle a, L_{1}^{\text {true }}\right)|\exists b\rangle L_{2},\left(L \rightarrow\left\langle a L_{1} b\right\rangle L_{2}\right) \in\right.\right.$ $P\} \cup\left\{\left(L^{\text {false }},\left\langle a, L_{1}^{\text {false }}\right) \mid\left(L \rightarrow\left\langle a L_{1}\right) \in P\right\}\right.\right.$

(3) the set of return edges, denoted as $\mathcal{M}_{\text {ret }}$, is defined as $\left\{\left(\left(L^{u}, L_{1}^{\text {true }}\right), b\right\rangle, L_{2}^{u}\right) \quad \mid \exists\langle a,(L \rightarrow$ $\left.\left.\left.\left.\left\langle a L_{1} b\right\rangle L_{2}\right) \in P\right\} \cup\left\{\left(L^{\text {false }}, b\right\rangle, L_{1}^{\text {false }}\right) \mid(L \rightarrow b\rangle L_{1}\right) \in P\right\}$.

Note that pending rules $L \rightarrow\left\langle a L_{1}\right.$ and $\left.L \rightarrow b\right\rangle L_{1}$ can be used only in edges starting with $L^{\text {false }}$; further, when using a matching rule $L \rightarrow\left\langle a L_{1} b\right\rangle L_{2}$ to generate edges starting with $L_{1}$, its tag must be true as $L_{1}$ should match only well-matched strings. As we will formalize later, a complete trace that constitutes a parse tree must satisfy the following constraints: (1) it must start with $L_{0}^{\text {false }}$, where $L_{0}$ is the start nonterminal, (2) it must end with some $L_{1}^{\text {false }}$ for some $L_{1}$ such that $\left(L_{1} \rightarrow \epsilon\right) \in P$; the tag must be false so that no matching rule is waiting to be finished, and $L_{1} \rightarrow \epsilon$ makes sure that no more inputs are expected to match $L_{1}$.

\footnotetext{
${ }^{2}$ For example, consider the grammar " $L \rightarrow \epsilon|c A| c B ; A \rightarrow d L ; B \rightarrow d L$ " and the string $w=(c d)^{n}$. The number of traces is $O\left(2^{n}\right)$.
} 
With the above definition of parse-tree edges, a parse tree is then a sequence of plain, call, or return edges. A parse forest is a sequence $\left[m_{1}, \ldots, m_{n}\right]$, where each $m_{i}$ is a subset of $\mathcal{M}_{\text {pln }}, \mathcal{M}_{\text {call }}$, or $\mathcal{M}_{\text {ret }}$. In the following discussion, we use $m_{\text {pln }}, m_{\text {call }}$, and $m_{\text {ret }}$ for an arbitrary subset of $\mathcal{M}_{\text {pln }}$, $\mathcal{M}_{\text {call }}$, and $\mathcal{M}_{\text {ret }}$, respectively.

Definition 5.2 (Parser PDA states and stack). Given a VPG, we introduce a parser PDA, where a state, denoted as $m$, is a subset of $\mathcal{M}_{\text {pln }}, \mathcal{M}_{\text {call }}$, or $\mathcal{M}_{\text {ret }}$, and each element in the stack $T$ is a subset of $\mathcal{M}_{\text {call }}$.

It is easy to see that a sequence of parser PDA states constitutes a parse forest.

Similar to the development of the VPG recognizer, we next define three derivative functions, denoted as $p_{c}, p_{\langle a}$, and $p_{b\rangle}$, to formalize how the parser PDA makes transitions. From the perspective of parse trees, each transition extends existing traces for parsing string $w$ to new traces for parsing string $w i$, assuming $i$ is the next input symbol. Notation-wise, we use the placeholder "_" to represent an entity whose value does not matter. For example, edge $\left({ }_{-}, L\right) \in m$ is defined as $\exists L_{1} i$, s.t. $\left(L_{1}, i, L\right) \in m$ or $\exists L_{1}, L_{2}, i, L$ s.t. $\left(\left(\left(L_{1}, L_{2}\right), i, L\right) \in m\right.$, where only $L$ is of interest.

Definition 5.3 (Derivative functions). Given a VPG $G=\left(V, \Sigma, P, L_{0}\right)$, suppose the current state of the parser PDA is $m$ and the current stack is $T$.

(1) $p_{c}(m)=\left(m^{\prime}, \lambda T . T\right)$, where $m^{\prime}=\left\{\left(L^{u}, c, L_{1}^{u}\right) \mid\left(,,, L^{u}\right) \in m \wedge\left(L \rightarrow c L_{1}\right) \in P\right\}$.

To generate $c$, we consider each parse-tree edge in $m$. If it is of the form $\left(,,, L^{u}\right)$, find every possible rule $L \rightarrow c L_{1}$ for some $L_{1}$. We then add edge $\left(L^{u}, c, L_{1}^{u}\right)$ to the next state $m^{\prime}$. Intuitively, if the current trace matches $w$ and the nonterminal to parse is $L$, then with the extra $c$, the new trace matches $w c$ and the new nonterminal to parse is $L_{1}$. Further, the boolean tag $u$ is passed from $L$ to $L_{1}$, since no matching rule is used at this step.

(2) $p_{\langle a}(m)=\left(m^{\prime}, \lambda T \cdot m^{\prime} \cdot T\right)$, where

$$
\begin{aligned}
m^{\prime}= & \left\{\left(L^{u},\left\langle a, L_{1}^{\text {true }}\right)\left|\left(,,, L^{u}\right) \in m \wedge \exists b\right\rangle L_{2},\left(L \rightarrow\left\langle a L_{1} b\right\rangle L_{2}\right) \in P\right\} \cup\right. \\
& \left\{\left(L^{\text {false }},\left\langle a, L_{1}^{\text {false }}\right) \mid\left(,,, L^{\text {false }}\right) \in m \wedge\left(L \rightarrow\left\langle a L_{1}\right) \in P\right\} .\right.\right.
\end{aligned}
$$

If $\left(,,-L^{\text {true }}\right) \in m$, a matching rule is waiting to be finished and we cannot use a pending rule such as $L \rightarrow\left\langle a L_{1}\right.$. Thus, only a new matching rule can be used to generate $\langle a$. So it finds every possible rule $L \rightarrow\left\langle a L_{1} b\right\rangle L_{2}$ and adds the parse-tree edge ( $L^{\text {true }},\left\langle a, L_{1}^{\text {true }}\right)$ to $m^{\prime}$. Notice that $L_{1}$ must be tagged with true. If $\left(\_, L^{\text {false }}\right) \in m$, either a matching or a pending rule can be used. The matching-rule case is similar to the case when $u=$ true. Further, it finds a rule like $L \rightarrow\left\langle a L_{1}\right.$ for some $L_{1}$ and adds ( $L^{\text {false }},\left\langle a, L_{1}^{\text {false }}\right.$ ) to $m^{\prime}$. In addition, the new state $m^{\prime}$ is pushed to the stack to match a possible return symbol at a later point.

(3) $p_{b\rangle}\left(m, m_{\text {call }}\right)=\left(m^{\prime}\right.$, tail $)$, where $m_{\text {call }}=$ head $T$ if $T \neq \perp$, and $m_{\text {call }}=\emptyset$ if $T=\perp$, and

$$
\begin{aligned}
m^{\prime}= & \left\{\left(\left(L^{u}, L_{1}^{\text {true }}\right), b\right\rangle, L_{2}^{u}\right) \mid\left(L^{u},\left\langle a, L_{1}^{\text {true }}\right) \in m_{\text {call }} \wedge \exists L_{2},\left(L \rightarrow\left\langle a L_{1} b\right\rangle L_{2}\right) \in P\right\} \cup \\
& \left.\left.\left\{\left(L^{\text {false }}, b\right\rangle, L_{1}^{\text {false }}\right) \mid\left(\_,, L^{\text {false }}\right) \in m \wedge(L \rightarrow b\rangle L_{1}\right) \in P\right\} .
\end{aligned}
$$

Consider $\left(,,, L^{u}\right) \in m$. If $u=$ false, we must use a pending rule to generate $\left.b\right\rangle$. Every rule $L \rightarrow b>L_{1}$ is converted to edge $\left.\left(L^{\text {false }}, b\right\rangle, L_{1}^{\text {false }}\right)$ and added to $m^{\prime}$. If $u=$ true, intuitively we can only use a matching rule to generate $b\rangle$. The information of the last unfinished matching rule is stored in $m_{\text {call }}$, the top of the stack. For any $\left(L^{u},\left\langle a, L_{1}^{\text {true }}\right) \in m_{\text {call }}\right.$, it finds a rule $L \rightarrow\left\langle a L_{1} b\right\rangle L_{2}$ for some $L_{2}$, and adds edge $\left.\left(\left(L^{u}, L_{1}^{\text {true }}\right), b\right\rangle, L_{2}^{u}\right)$ to the new state $m^{\prime}$. The nonterminal $L_{2}$ inherits its tag from the tag of $L$. Note that in the above formulation the new state is generated based on only $m_{\text {call }}$, not the current state $m$; this design can generate invalid edges; a later pruner step will remove those invalid edges. 


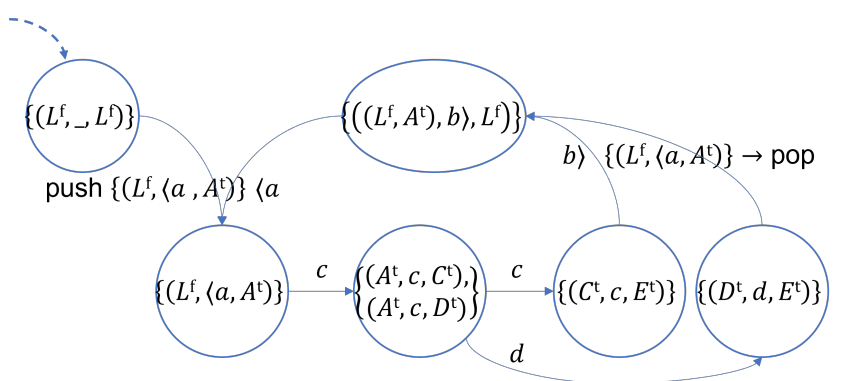

Fig. 5. The parser PDA for the grammar in Figure 2. We use $\mathrm{f}$ for false and $\mathrm{t}$ for true. Finally, the symbols and stack actions of $\langle a$ and $b\rangle$ are each shared by two transitions.

Constructing the parser PDA. Similar to constructing the recognizer PDA, the construction of the parser PDA is the least solution of the following equation.

$$
\begin{aligned}
A= & A \cup\left\{m^{\prime} \mid c \in \Sigma_{l} \wedge m \in A \wedge p_{c}(m)=\left(m^{\prime}, f\right)\right\} \\
& \cup\left\{m^{\prime} \mid\left\langle a \in \Sigma_{c} \wedge m \in A \wedge p_{\langle a}(m)=\left(m^{\prime}, f\right)\right\}\right. \\
& \cup\left\{m^{\prime}|b\rangle \in \Sigma_{r} \wedge m \in A, m^{\prime \prime} \in\left(A \cap \mathcal{P}\left(\mathcal{M}_{\text {call }}\right)\right) \cup\{\emptyset\} \wedge p_{b\rangle}\left(m, m^{\prime \prime}\right)=\left(m^{\prime}, f\right)\right\}
\end{aligned}
$$

Different from the case for VPG recognizers, $\left(A \cap \mathcal{P}\left(\mathcal{M}_{\text {call }}\right)\right) \cup\{\emptyset\}$ is used for $p_{b\rangle}$, since a stack element for the parser PDA has to be a state generated for a call symbol and the stack can also be empty.

With the above equation, we can construct an algorithm for computing all parser PDA states and state transitions, similar to Algorithm 1. The differences are that (1) it starts with a helper state $m_{0}=\left\{\left(L_{0}^{\text {false }},{ }_{-}, L_{0}^{\text {false }}\right)\right\}$, where $L_{0}$ is the start nonterminal of the input VPG and _ stands for a dummy nonterminal, and (2) it uses parser derivative functions for deriving new states and transitions. The result is a parser PDA whose states are subsets of $\mathcal{M}_{\text {pln }}, \mathcal{M}_{\text {call }}$, or $\mathcal{M}_{\text {ret }}$, and transitions between states are labeled with $(c, f),\left(\langle a, f)\right.$, or $\left.(b\rangle, m_{c}, f\right)$, where $c,\langle a$, or $b\rangle$ is the next input symbol, $m_{c}$ is the top stack element, and $f$ is the stack action. Figure 5 shows the parser PDA generated by the algorithm for the grammar in Figure 2.

Given an input string, the parser PDA starts from $\left(m_{0}, \perp\right)$ and transitions to the next runtime configuration based on the following definition.

Definition 5.4 (Runtime transition for parser PDA). Suppose the current configuration is $(m, T)$ and the next input symbol is $i$. The runtime transition function $\mathcal{P}(i, m, T)=\left(m^{\prime}, T^{\prime}\right)$ of the parser PDA is defined as follows.

(1) if $i \in \Sigma_{c} \cup \Sigma_{l}$ and PDA edge $\left(m, m^{\prime}\right)$ is marked with $(i, f)$, then $\mathcal{P}(i, m, T)=\left(m^{\prime}, f(T)\right)$;

(2) if $i \in \Sigma_{r}, T=m^{\prime \prime} \cdot T^{\prime}$, and PDA edge $\left(m, m^{\prime}\right)$ is marked with $\left(i, m^{\prime \prime}, f\right)$, then $\mathcal{P}(i, m, T)=$ $\left(m^{\prime}, f(T)\right)$.

(3) if $i \in \Sigma_{r}, T=\perp$, and PDA edge $\left(m, m^{\prime}\right)$ is marked with $(i, \emptyset, f)$, then $\mathcal{P}(i, m, T)=\left(m^{\prime}, \perp\right)$.

\subsection{The Pruner PDA and the Extractor}

The parser PDA parses an input string $w$ of length $n$ and produces a state trace: $\left[m_{1}, \ldots, m_{n}\right]$, which can be viewed as a parse forest. As discussed earlier, it can contain invalid edges. For example, inside the last state $m_{n}$, a valid edge must be of the form $\left({ }_{-}, L_{1}^{\text {false }}\right)$ for some $L_{1}$ so that $\left(L_{1} \rightarrow \epsilon\right) \in P$, signaling the end of parsing; other edges are invalid. Similarly, if the next symbol to match is a return symbol produced in a matching rule $L \rightarrow\left\langle a L_{1} b\right\rangle L_{2}$, the parse-tree edge immediately before the one that produces the return symbol must end with $L_{1}^{\text {false }}$ so that $\left(L_{1} \rightarrow \epsilon\right) \in P$. 


$$
\begin{gathered}
\frac{(L \rightarrow \epsilon) \in P}{L^{u} \Rightarrow(\epsilon,[])} \quad \frac{\left(L \rightarrow c L_{1}\right) \in P \quad L_{1}^{u} \Rightarrow\left(w_{1}, v_{1}\right)}{L^{u} \Rightarrow\left(c w_{1},\left(L^{u}, c, L_{1}^{u}\right):: v_{1}\right)} \\
\frac{\left(L \rightarrow\left\langle a L_{1}\right) \in P \quad L_{1}^{\text {false }} \Rightarrow\left(w_{1}, v_{1}\right)\right.}{L^{\text {false }} \Rightarrow\left(\left\langlea w_{1},\left(L^{\text {false }},\left\langle a, L_{1}^{\text {false }}\right):: v_{1}\right)\right.\right.} \quad \frac{\left.(L \rightarrow b\rangle L_{1}\right) \in P \quad L_{1}^{\text {false }} \Rightarrow\left(w_{1}, v_{1}\right)}{\left.\left.L^{\text {false }} \Rightarrow(b\rangle w_{1},\left(L^{\text {false }}, b\right\rangle, L_{1}^{\text {false }}\right):: v_{1}\right)} \\
\frac{\left(L \rightarrow\left\langle a L_{1} b\right\rangle L_{2}\right) \in P \quad L_{1}^{\text {true }} \Rightarrow\left(w_{1}, v_{1}\right) \quad L_{2}^{u} \Rightarrow\left(w_{2}, v_{2}\right)}{L^{u} \Rightarrow\left(\left\langle a w_{1} b\right\rangle w_{2},\left[\left(L^{u},\left\langle a, L_{1}^{\text {true }}\right)\right]+v_{1}+\left[\left(\left(L^{u}, L_{1}^{\text {true }}\right), b\right\rangle, L_{2}^{u}\right)\right]+v_{2}\right)} .
\end{gathered}
$$

Fig. 6. The big-step parse-tree derivation, assuming a VPG $G=\left(\Sigma, V, P, L_{0}\right)$.

After removing some invalid edges, earlier edges in the parse forest may become invalid. For instance, if pruning removes from $m_{n}$ an edge $\left(L_{2}^{\text {false }}, \ldots,-\right)$ and there are no other edges that start with $L_{2}^{\text {false }}$ in the rest of $m_{n}$, then any $m_{n-1}$ edge that ends with $L_{2}^{\text {false }}$ can also be pruned, since it is not possible to connect it with an edge in the pruned $m_{n}$. This is a backward process. Therefore, our pruner PDA takes the reverse of the parse forest, $\left[m_{n}, m_{n-1}, \ldots, m_{1}\right]$, as the input and produces a pruned parse forest $\left[m_{n}^{\prime}, m_{n-1}^{\prime}, \ldots, m_{1}^{\prime}\right]$ in reverse. Further, instead of intervening parsing and pruning steps, we choose to perform pruning after the parser PDA has finished so that every state in the parse forest gets pruned only once.

Definition 5.5 (Pruner PDA states and stack). Given a VPG, we introduce a pruner PDA, where each state, denoted as $m$, is a subset of $\mathcal{M}_{\text {pln }}, \mathcal{M}_{\text {call }}$ or $\mathcal{M}_{\text {ret }}$, and each element of the stack $T$ is a subset of $\mathcal{M}_{\text {ret }}$.

A stack is needed in the pruner to find valid call edges with respect to valid return edges in the stack. The technical details of the pruner PDA construction are introduced in Appendix C of the companion technical report (Jia et al. 2021). After pruning, we get a pruned parse forest $\left[m_{1}^{\prime}, \ldots, m_{n}^{\prime}\right]$ and an extractor is then used to extract parse trees from the forest. If the input VPG is unambiguous, at most one parse tree can be extracted. The definition of extract $\left(\left[m_{1}^{\prime}, \ldots, m_{n}^{\prime}\right]\right)$ detailed in Appendix $\mathrm{C}$ of the companion technical report (Jia et al. 2021) extracts a parse-tree set $V$, which is a set of parse trees together with corresponding stacks of call edges.

\subsection{The Correctness Proof of the Parsing Algorithm}

In this section, we discuss the correctness proof of our core VPG parsing algorithm; the proof is formalized in the proof assistant Coq. The correctness theorem is stated based on the relation $L^{u} \Rightarrow(w, v)$, meaning that input string $w$ can be parsed from nonterminal $L$ with tag $u$ and one of the parse trees is $v$. We call this relation the big-step parse-tree derivation relation, which is presented in Figure 6. Its rules are mostly straightforward and we explain only the one for $L \rightarrow\left\langle a L_{1} b\right\rangle L_{2}$ : it first builds a parse tree for substring $w_{1}$ with $L_{1}^{\text {true }}$ since $w_{1}$ must be a well-matched string; it then builds a parse tree for substring $w_{2}$ with $L_{2}^{u}$; then a parse tree for string $\left\langle a w_{1} b\right\rangle w_{2}$ can be built by concatenating the parse-tree edge for $\left\langle a\right.$, the parse tree for $w_{1}$, the parse-tree edge for $\left.b\right\rangle$, and the parse tree for $w_{2}$.

Definition 5.6. Suppose $v$ is a trace of parse-tree edges. We define firstNT(v) to be the starting nonterminal in the trace and lastNT $(v)$ to be the last nonterminal in the trace. That is,

$$
\begin{aligned}
& \operatorname{firstNT}(v)=\left\{L^{u} \mid v=\left(L^{u},,_{-}\right)::_{-}\right\} \\
& \operatorname{lastNT}(v)=\left\{L^{u} \mid v==_{-}+\left[\left({ }_{-}, L^{u}\right)\right]\right\}
\end{aligned}
$$




$$
\begin{aligned}
& \frac{\left(L \rightarrow c L_{1}\right) \in P \quad v=[] \vee \operatorname{lastNT}(v)=L^{u}}{(v, E) \stackrel{c}{\rightarrow}\left(v+\left[\left(L^{u}, c, L_{1}^{u}\right)\right], E\right)} \\
& \left(L \rightarrow\left\langle a L_{1}\right) \in P \quad v=[] \vee \operatorname{lastNT}(v)=L^{\text {false }}\right. \\
& (v, E) \stackrel{\langle a}{\rightarrow}\left(v+\left[\left(L^{\text {false }},\left\langle a, L_{1}^{\text {false }}\right)\right],\left(L^{\text {false }},\left\langle a, L_{1}^{\text {false }}\right) \cdot E\right)\right.\right. \\
& \frac{\left(L \rightarrow\left\langle a L_{1} b\right\rangle L_{2}\right) \in P \quad v=[] \vee \operatorname{lastNT}(v)=L^{u}}{(v, E) \stackrel{\langle a}{\rightarrow}\left(v+\left[\left(L^{u}\left\langle a, L^{\text {true }}\right)\right]\left(L^{u},\left\langle a, L^{\text {true }}\right) \cdot E\right)\right.\right.} \\
& \left.(L \rightarrow b\rangle L_{1}\right) \in P \quad v=[] \vee \operatorname{lastNT}(v)=L^{\text {false }} \\
& \left.(v, \perp) \stackrel{b\rangle}{\rightarrow}\left(v+\left[\left(L^{\text {false }}, b\right\rangle, L_{1}^{\text {false }}\right)\right], \perp\right) \\
& \left(L \rightarrow\left\langle a L_{1} b\right\rangle L_{2}\right) \in P \quad \operatorname{lastNT}(v)=L_{3}^{\text {true }} \quad\left(L_{3} \rightarrow \epsilon\right) \in P \\
& \left(v,\left(L^{u},\left\langle a, L_{1}^{\text {true }}\right) \cdot E\right) \stackrel{b\rangle}{\rightarrow}\left(v+\left[\left(\left(L^{u}, L_{1}^{\text {true }}\right), b\right\rangle, L_{2}^{u}\right)\right], E\right) \\
& \left(L_{3} \rightarrow\left\langle a L_{4}\right) \in P \quad\left(L_{1} \rightarrow b\right\rangle L_{2}\right) \in P \quad v=[] \vee \operatorname{lastNT}(v)=L_{1}^{\text {false }} \\
& \left(v,\left(L_{3}^{\text {false }},\left\langle a, L_{4}^{\text {false }}\right) \cdot E\right) \stackrel{b\rangle}{\rightarrow}\left(v+\left[\left(L_{1}^{\text {false }}, b\right\rangle, L_{2}^{\text {false }}\right)\right], E\right)
\end{aligned}
$$

Fig. 7. The small-step parse-tree derivation, given a VPG $G=\left(\Sigma, V, P, L_{0}\right)$.

$$
\frac{\left(v_{1}, E_{1}\right) \stackrel{w}{\longrightarrow} *\left(v_{2}, E_{2}\right) \quad\left(v_{2}, E_{2}\right) \stackrel{i}{\rightarrow}\left(v_{3}, E_{3}\right)}{(v, E) \stackrel{\epsilon}{\longrightarrow} *(v, E)}
$$

Fig. 8. The transitive closure of the small-step.

Theorem 5.1 (Correctness of VPG Parsing). For an input string $w$ of length $n$, if the parser $P D A$ for $V P G G=\left(\Sigma, V, P, L_{0}\right)$ starts with the initial configuration $m_{0}=\left\{\left(L_{0}^{\text {false }},{ }_{-}, L_{0}^{\text {false }}\right)\right\}$ and $T_{0}=\perp$ and traverses the following configurations to parse $w:\left(m_{0}, T_{0}\right) \stackrel{w_{1}}{\longrightarrow}\left(m_{1}, T_{1}\right) \cdots \stackrel{w_{n}}{\longrightarrow}\left(m_{n}, T_{n}\right)$, and $\operatorname{extract}\left(\left[m_{1}, \ldots, m_{n}\right]\right)=V$, then

$$
\forall v, L_{0}^{\text {false }} \Rightarrow(w, v) \text { iff }\left(\exists E,(v, E) \in V \wedge \exists L_{1}, \operatorname{lastNT}(v)=L_{1}^{\text {false }} \wedge\left(L_{1} \rightarrow \epsilon\right) \in P\right) .
$$

To prove the theorem, we need the help of a small-step parse-tree derivation relation so that we can formalize a set of invariants that are satisfied during each step when running the parser PDA. The relation $(v, E) \stackrel{i}{\rightarrow}\left(v^{\prime}, E^{\prime}\right)$, defined in Figure 7 , means that starting with a parse tree $v$ and a stack of call edges $E$, the parsing of symbol $i$ results in a new parse tree $v^{\prime}$ and a new stack of call edges $E^{\prime}$. In all rules, $v^{\prime}$ is the result of adding one new parse-tree edge to $v$, and therefore it formalizes the process of generating one parse-tree edge at a time, matching what the parser PDA does. The transitive closure of the small-step relation is in Figure 8. The following two theorems show the equivalence of big-step and small-step parse-tree relations.

Theorem 5.2 (From Big STEP TO SMALl STEP). 
(1) If $L^{\text {true }} \Rightarrow(w, v)$, then $([], \perp) \stackrel{w}{\longrightarrow} *(v, \perp)$.

(2) If $L^{\text {false }} \Rightarrow(w, v)$, then $\exists E$, s.t. $([], \perp) \stackrel{w}{\longrightarrow} *(v, E)$.

Theorem 5.3 (From SMAll STEP TO Big Step). If $([], \perp) \stackrel{w}{\longrightarrow} *(v, E)$, and firstNT $(v)=L^{f a l s e}$, lastNT $(v)=L_{1}^{\text {false }}$ and $\left(L_{1} \rightarrow \epsilon\right) \in P$, then $L^{\text {false }} \Rightarrow(w, v)$.

With the small-step relation, we can formalize those invariants satisfied by every step when running the parser PDA. Suppose the parser PDA has consumed string $w^{\prime}$ to reach configuration $\left(m^{\prime}, T^{\prime}\right)$ and consumes symbol $i$ next to reach $(m, T)$. Further, the extractor (Definition C.7 in Appendix $\mathrm{C}$ of the companion technical report (Jia et al. 2021)) can extract a parse-tree set $V^{\prime}$ from the parser PDA state traces for $w^{\prime}$. Finally, extending $V^{\prime}$ with one-more parse-tree edge in $m$ to get $V$. Then the following theorem can be proved: if the previous step satisfies $\operatorname{Inv}\left(L, w^{\prime}, m^{\prime}, T^{\prime}, V^{\prime}\right)$, the latest step should also satisfy $\operatorname{Inv}(L, w, m, T, V)$, where $w=w^{\prime} i$. The invariants are defined as follows.

Property 5.4 (InVARIANTS OF VPG PARSING). Let $L$ be a nonterminal, $w$ an input string, $(m, T) a$ parser PDA configuration, and $V$ a parse-tree set. The property $\operatorname{Inv}(L, w, m, T, V)$ is defined as

(1) $\forall v E,(v, E) \in V$ if and only if $([], \perp) \stackrel{w}{\longrightarrow} *(v, E) \wedge$ firstNT $(v)=L$;

(2) $\forall(v, E) \in V,(E=\perp \rightarrow T=\perp) \wedge\left(\exists e E^{\prime}, E=e \cdot E^{\prime} \rightarrow e \in\right.$ head $\left.T\right)$;

(3) $\forall v, \exists E,(v, E) \in V \rightarrow \exists e, e \in m \wedge v=_{-}+[e]$.

The above correctness proof is formalized in Coq and includes around 3k lines of proofs for the correspondence between the big-step and the small-step parse-tree derivations and another $4 \mathrm{k}$ lines for implementing the parser and the parse-tree extractor, formalizing the invariants, and proofs for showing that the invariants are preserved during parsing.

\subsection{Time and Space Complexity}

When given an input of length $n$, the VPG parser runs two PDAs to construct the parse forest: a forward parser PDA and a backward pruner PDA. We assume their transition tables can be implemented via a data structure that provides constant-time lookups (e.g., via a hash table) Therefore, each transition can finish in constant time, leading to the linear-time running of VPG parsing.

The space complexity depends on the space for representing the transition tables of the two PDAs. Recall that the transition function of the parser PDA is $\mathcal{P}(i, m, T)=\left(m^{\prime}, T^{\prime}\right)$, where $i \in \Sigma, m$ and $m^{\prime}$ are states, and $T$ and $T^{\prime}$ are stacks. The transition function of the pruner PDA is $\mathcal{G}\left(m_{1}, m_{2}^{\prime}, T\right)=$ $\left(m_{1}^{\prime}, T^{\prime}\right)$, where $m_{1}, m_{2}^{\prime}$, and $m_{1}^{\prime}$ are states, and $T$ and $T^{\prime}$ are stacks of states. In fact, only the top of the stack is used by $\mathcal{P}$ and $\mathcal{G}$. Note since a state is a set of edges and an edge corresponds to a rule, the size of a state is at most $O(|P|)$. Thus, there are at most $O\left(2^{|P|}\right)$ states. So the total number of transitions in the two PDAs is bounded by $O\left(2^{3|P|} \times|\Sigma|+2^{4|P|}\right)$, where $\Sigma$ is the input alphabet. Each entry in the transition table occupies $O(\log |\Sigma|+|P|)$ bits. As a conclusion, the space complexity is $O\left((\log |\Sigma|+|P|) \times\left(2^{3|P|} \times|\Sigma|+2^{4|P|}\right)\right)$, which is exponential in $|P|$. However, this is the worst-case scenario as not all states can be derived; further, it is independent of the input string size.

In our evaluation, the largest space occupied by the transition tables is around 1.6 MB, for an HTML grammar (discussed in Section 7.3).

\section{DESIGNING A SURFACE GRAMMAR}

The format of rules allowed in VPGs is designed for easy studying of its meta-theory, but is inconvenient for expressing practical grammars. First, no user-defined semantic actions are allowed. 
Second, each VPG rule allows at most four terminals/nonterminals on the right-hand side. In this section, we present a surface grammar that is more user-friendly for writing grammars. We first discuss embedding semantic actions. Then we introduce tagged CFGs, which are CFGs paired with information about how to separate terminals to plain, call, and return symbols. We then describe a translator from tagged CFGs to VPGs. During the conversion, the translator also generates semantic actions that convert the parse trees of VPGs back to the ones of tagged CFGs.

\subsection{Embedding Semantic Actions}

Semantic actions transform parsing results to user-preferred formats. In a rule $L \rightarrow s_{1} \cdots s_{k}$, where $s_{k} \in \Sigma \cup V$, we treat $L$ as a default action that takes $k$ arguments, which are semantic values returned by $s_{1}$ to $s_{k}$, and returns a tree with a root node and $s_{1}$ to $s_{k}$ as children. The prefix notation of a parse tree gives

$$
\left[L, v_{s_{1}}, \cdots, v_{s_{k}}\right]
$$

where $v_{s_{i}}$ is the semantic value for $s_{i}$. The above notation can be naturally viewed as a stack machine, where $L$ is an action and $v_{s_{i}}$ are the values that get pushed to the stack before the action. The VPG parse tree can be converted to the prefix notation in a straightforward way. If we then replace each nonterminal in the tree with its semantic action, the parse tree becomes a stack machine.

The default action for a nonterminal can be replaced by a user-defined action appended to each rule in the grammar. For example, consider the grammar $L=c L|\langle a L b\rangle L| \epsilon$. Suppose we want to count the number of the symbol $c$ in an input string; we can specify semantic actions in the grammar as follows.

$$
L \rightarrow c L @\left\{\text { let } f_{1} v_{1} v_{2}=1+v_{2}\right\} \mid\langle a L b\rangle L @\left\{\text { let } f_{2} v_{1} v_{2} v_{3} v_{4}=v_{2}+v_{4}\right\} \mid \epsilon @\left\{\text { let } f_{3}()=0\right\} .
$$

In the above example, a semantic action is specified after each rule, e.g., "@ $\left\{\right.$ let $\left.f_{1} v_{1} v_{2}=1+v_{2}\right\}$ ". In the actions, $v_{1}, v_{2}, v_{3}$ and $v_{4}$ represent the semantic values returned by the right hand side symbols of the rule. For example, the first semantic action $f_{1} v_{1} v_{2}=1+v_{2}$ accepts two semantic values $v_{1}$ and $v_{2}$, where $v_{1}$ is returned by $c$ and $v_{2}$ is returned by $L$.

As an application, the next subsection shows how to use semantic actions to convert the parse trees of a VPG to the parse trees of its original tagged CFG.

\subsection{Translating from Tagged CFGs to VPGs}

Grammar writers are already familiar with CFGs, the basis of many parsing libraries. We define tagged CFGs to be CFGs paired with information about how to partition terminals into plain, call, and return symbols $\left(\Sigma=\Sigma_{l} \cup \Sigma_{c} \cup \Sigma_{r}\right) ;^{3}$ that is, in a tagged CFG, a terminal is tagged with information about what kind of symbols it is. Compared to a regular CFG, the only additional information in a tagged CFG is the tagging information; therefore, tagged CFGs provide a convenient mechanism for reusing existing CFGs and developing new grammars in a mechanism that grammar writers are familiar with. Appendix D of the companion technical report (Jia et al. 2021) shows some example tagged CFGs.

However, not all tagged CFGs can be converted to VPGs. We use a conservative validator to determine if a tagged CFG can be converted to a VPG and, if the validator passes, translate the tagged CFG to a VPG. For simplicity, we assume every call symbol is matched with a return symbol in the input tagged CFG.

The translation steps are summarized as follows:

$$
\text { A tagged } C F G \rightarrow \text { Simple form } \stackrel{\text { If valid }}{\longrightarrow} \text { Linear form } \rightarrow \text { VPG. }
$$

\footnotetext{
${ }^{3}$ We note our implementation of tagged CFGs additionally supports regular operators in the rules; these regular operators can be easily desugared and we omit their discussion.
} 
At a high level, a tagged CFG is first translated to a simple form, upon which validation is performed. If validation passes, the simple-form CFG is translated to a linear-form CFG, which is finally translated to a VPG. We next detail these steps.

Definition 6.1 (Simple forms). A rule is in the simple form if it is of the form $L \rightarrow \epsilon$, or of the form $L \rightarrow s_{1} \cdots s_{k}$, where $s_{i} \in \Sigma_{l} \cup V$ or $\exists\langle a, b\rangle, L_{i}$, s.t. $s_{i}=\left\langle a L_{i} b\right\rangle, i=1 . . k, k \geq 1$. A tagged CFG $G=\left(V, \Sigma, P, L_{0}\right)$ is in the simple form, if every rule in $P$ is in the simple form.

Compared to a tagged CFG, a simple-form CFG requires that there must be a nonterminal between a call symbol and its matching return symbol. The conversion from a tagged CFG to a simple-form CFG is straightforward: for each rule, we replace every string $\langle a s b\rangle$, where $\langle a$ is matched with $b\rangle$ and $s \in(\Sigma \cup V)^{*}$, with $\left\langle a L_{s} b\right\rangle$ and generate a new nonterminal $L_{s}$ and a new rule $L_{s} \rightarrow s$. After this conversion, a string in the from of $\langle a L b\rangle$ can be viewed as a "plain symbol"; this is a key intuition for the following steps. We call $\langle a L b\rangle$ a matched token in the following discussion.

The validation can then perform on the simple form, using its dependency graph.

Definition 6.2 (Dependency graphs). The dependency graph of a grammar $G=\left(V, \Sigma, P, L_{0}\right)$ is $\left(V, E_{G}\right)$, where $E_{G}=\left\{\left(L, L^{\prime}\right) \mid \exists s_{1}, s_{2} \in(\Sigma \cup V)^{*}\right.$, s.t. $\left.\left(L \rightarrow s_{1} L^{\prime} s_{2}\right) \in P\right\}$.

The validator checks for every loop in the dependency graph, either (1) in the loop there is an edge $\left(L, L^{\prime}\right)$ that is produced from a rule of the form $L \rightarrow s_{1}\left\langle a L^{\prime} b\right\rangle s_{2}$, where $s_{1}, s_{2} \in(\Sigma \cup V)^{*}$; or (2) every edge $\left(L, L^{\prime}\right)$ in the loop is produced from a rule of the form $L \rightarrow s L^{\prime}, s \in(\Sigma \cup V)^{*}$ and at least one edge in the loop satisfies $s \nrightarrow^{*} \epsilon$.

Once the validation passes, the translation converts a simple-form CFG to a linear-form CFG.

Definition 6.3 (Linear forms). A rule is in the linear form if it is in one of the following forms: (1) $L \rightarrow \epsilon$; (2) $L \rightarrow t_{1} \cdots t_{k}$; (3) $L \rightarrow t_{1} \cdots t_{k} L^{\prime}$; where $t_{i} \in \Sigma_{l}$ or $\exists\langle a, b\rangle$, $L_{i}$, s.t. $t_{i}=\left\langle a L_{i} b\right\rangle, i=1 . . k$, $k \geq 1$. A tagged CFG $G=\left(V, \Sigma, P, L_{0}\right)$ is in the linear form if every rule in $P$ is in the linear form.

Note that in a linear-form rule, $t_{i}$ cannot be a nonterminal, while in a simple-form rule $s_{i}$ can be a nonterminal. Further, the linear form allows rules of the form $L \rightarrow t_{1} \cdots t_{k} L^{\prime}$, where $t_{i}$ is a terminal or a matched token. The main job of the translator is to convert simple-form rules to linear-form rules. Appendix E of the companion technical report (Jia et al. 2021) shows the translation algorithm.

The translation from a linear-form CFG to a VPG is simple. E.g., for a rule of the form $L \rightarrow t_{1} \cdots t_{k}$, it is translated to $L \rightarrow t_{1} L_{1} ; L_{1} \rightarrow t_{2} L_{2} ; \ldots ; L_{k} \rightarrow t_{k} L_{k} ; L_{k} \rightarrow \epsilon$, where $L_{1}$ to $L_{k}$ are a set of new nonterminals.

All transformations are local rewriting of rules and as a result it is easy to show that each transformation step preserves the set of strings the grammar accepts. We further note that not all tagged CFGs can be converted to VPGs. For example, grammar " $L \rightarrow c L c \mid \epsilon$ " cannot be converted since its terminals cannot be suitably tagged: intuitively $c$ has to be both a call and a return symbol. Further, since our validation algorithm is conservative, it rejects some tagged CFGs that have VPG counterparts. For example, grammar " $L \rightarrow L c \mid \epsilon$ " is rejected by the validator since it is left recursive. However, it can be first refactored to " $L \rightarrow c L \mid \epsilon$ ", which is accepted by our validator.

Generating semantic actions. During the conversion, each time the translator rewrites a rule, a corresponding semantic action is attached to the rule. Initially, every rule is attached with one default semantic action. For example, the rule $L \rightarrow A b C d$ is attached with $L^{4}$, written as $L \rightarrow A b C d @ L^{4}$. As mentioned in Section 6.1, $L^{4}$ is the default semantic action for constructing a tree with a root node and children nodes that are constructed from semantic values from the right hand side of the rule. The superscript 4 is its arity. During conversion, every time we rewrite a nonterminal $L$ 
in a rule $R$ with the right-hand side of rule $L \rightarrow s$, the semantic values for $s$ are first combined to produce a semantic value for $L$, which is then used to produce the semantic value for the left-hand nonterminal of $R$. If a helper nonterminal $L_{s}$ is introduced during conversion and a rule $L_{s} \rightarrow s$ is generated, we do not generate a semantic value for $L_{s}$ but leave the semantic values for $s$ on the stack so that any rule that uses $L_{s}$ can use those semantic values directly. In this way, we can convert a parse tree of a VPG to the parse tree of its corresponding tagged CFG. Appendix F of the companion technical report (Jia et al. 2021) shows an example of the translation.

\section{EVALUATION}

We implemented our VPG parsing library in OCaml. The implementation used hash tables to store the transition tables of the generated parser and pruner PDAs to get constant-time lookup. We evaluated our implementation for the following questions: (1) how applicable VPG parsing is in practice? (2) what is the performance of VPG parsing compared with other parsing approaches?

We performed a preliminary analysis for a set of ANTLR4 grammars in a grammar repository ${ }^{4}$. Among all 239 grammars, 136 (56.9\%) grammars could be converted to VPGs by our tagged-CFGto-VPG translation, after we manually marked the call and return symbols for those grammars. Note that it does not mean the rest cannot be converted; e.g., 34 grammars cannot be converted because they have left recursion and the conversion may become possible if the left recursion is removed. We left a further analysis for future work.

For performance evaluation, we compared our VPG parsers with ANTLR4 ${ }^{5}$, a popular parser generator that implements an efficient parsing algorithm called ALL $\left({ }^{*}\right)$ (Parr et al. 2014). The $\operatorname{ALL}\left({ }^{*}\right)$ algorithm can perform an unlimited number of lookaheads to resolve ambiguity and it has a worst-case complexity of $O\left(n^{4}\right)$; however, it exhibits linear behavior on many practical grammars. We also compared the VPG parsers with a few hand-crafted parsers specialized for parsing JSON and XML documents, including four mainstream JavaScript engines and four popular XML parsers. Before presenting the performance evaluation, we list some general setups:

(1) During evaluation, we adapted the grammars for JSON, XML, and HTML from ANTLR4 ${ }^{6}$ to tagged CFGs, generated VPG parsers, and compared VPG parsers with the parsers generated by ANTLR in performance. Appendix D of the companion technical report (Jia et al. 2021) shows the tagged CFGs for JSON, XML and HTML.

(2) When comparing with ANTLR, we compared only the parsing time, omitting the lexing time. This is because we used ANTLR's lexers to generate the tokens for both VPG parsers and ANTLR parsers.

\subsection{Comparison with ANTLR on Parsing JSON Files}

The JSON format allows objects to be nested within objects and arrays; therefore, a JSON object has a hierarchically nesting structure, which can be naturally captured by a VPG. In particular, since in JSON an object is enclosed within "\{" and "\}" and arrays within "[" and "]", its VPG grammar treats "\{" and "[" as call symbols and treats "\}" and "]" as return symbols.

When building a VPG parser for JSON, we reused ANTLR's lexer. Therefore, the evaluation steps are as follows.

$$
\text { Input file } \stackrel{\text { ANTLR Lexer }}{\longrightarrow} \text { ANTLR tokens } \stackrel{\text { ANTLR Parser or VPG Parser }}{\longrightarrow} \text { Results. }
$$

\footnotetext{
${ }^{4}$ https://github.com/antlr/grammars-v4

${ }^{5}$ https://www.antlr.org/.

${ }^{6}$ https://github.com/antlr/grammars-v4/blob/master/json/, https://github.com/antlr/grammars-v4/blob/master/xml/, and https://github.com/antlr/grammars-v4/tree/master/html
} 


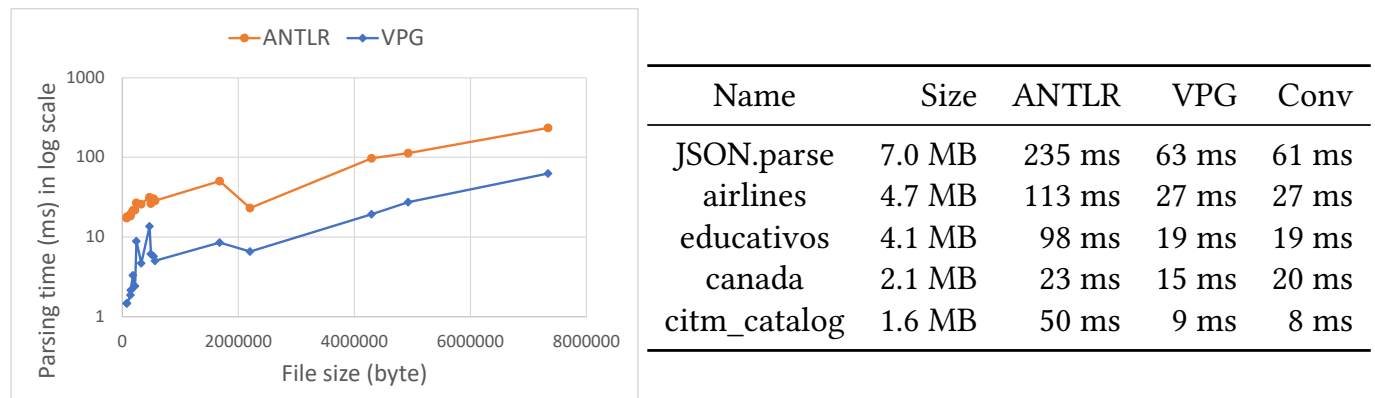

Fig. 9. Parsing times of JSON files (in log scale on the left).

For evaluation, we collected 23 real-world JSON files from the awesome-json repository, the nativejson benchmarks, and the JSON. parse benchmarks ${ }^{7}$. The sizes of the files range from $14 \mathrm{~KB}$ to $7 \mathrm{MB}$. The parsing times are shown in Figure 9; note that the y-axis of the left figure (and other figures in this section) is in the log scale for better visualization. As can be seen, the VPG parser runs much faster than ANTLR. The right of Figure 9 shows the VPG parsing times for the 5 largest files in our test set; the VPG column is the amount of time cost by running the parser and pruner PDAs. On those large files, VPG parsing is about 4 times faster than ANTLR. For smaller files, the gap is even larger; Appendix G of the companion technical report (Jia et al. 2021) shows the results for the full test set.

A downstream application that uses the ANTLR's JSON parser may wish to keep working on the same parsing result produced by ANTLR's parser. Therefore, we implemented a converter to convert the parse forest produced by our VPG parser to ANTLR's parse tree for the input files. When the grammar is unambiguous, which is the case for the JSON grammar (as well as the XML and HTML grammars), the parse forest is really the encoding of a single parse tree. The algorithm of how to convert a VPG parse tree to a stack machine and how to evaluate the stack machine have been discussed in Section 6. The result of the evaluation is a structure that can be directly printed out and compared with; the same applies to the ANTLR parse tree ${ }^{8}$. The conversion steps are summarized as follows.

$$
\text { VPG parse tree } \stackrel{\text { Embed actions }}{\longrightarrow} \text { Stack machine } \stackrel{\text { Evaluate }}{\longrightarrow} \text { ANTLR parse tree. }
$$

Note that in practice this conversion may not be necessary. A downstream application can directly work on the VPG parse tree. We include the time to show the conversion time for our VPG parser to work directly with legacy downstream applications. The time of conversion is shown in the "Conv" column on the right hand side of Figure 9.

\subsection{Comparison with ANTLR on Parsing XML Files}

XML also has a well-matched nesting structure with explicit start-tags such as $\langle p\rangle$ and matching end-tags such as $</ p>$. However, compared to JSON, there is an additional complexity for the XML grammar, which makes it necessary to adapt the XML grammar provided by ANTLR. In particular, the XML lexer in ANTLR treats an XML tag as separate tokens; e.g., $\langle p\rangle$ is converted into three tokens: $<, p$, and $>$. Those tokens then appear in the ANTLR XML grammar. Part of the reason for this design is because the XML format allows additional attributes within a tag; e.g., $\langle p \quad i d=1>$

\footnotetext{
${ }^{7}$ https://github.com/jdorfman/awesome-json-datasets, https://github.com/miloyip/nativejson-benchmark, and https:// github.com/GoogleChromeLabs/json-parse-benchmark

${ }^{8}$ By the "ANTLR parse tree", we mean the string output by the ANTLR parser with the option "-tree".
} 


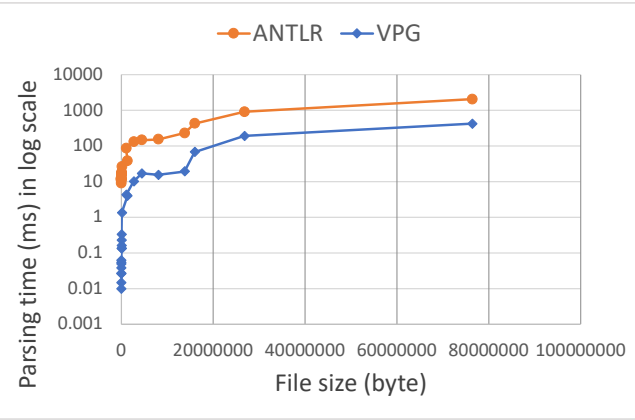

\begin{tabular}{crrrr}
\hline Name & Size & ANTLR & VPG & Conv \\
\hline po & $73 \mathrm{MB}$ & $2058 \mathrm{~ms}$ & $425 \mathrm{~ms}$ & $1070 \mathrm{~ms}$ \\
cd & $26 \mathrm{MB}$ & $913 \mathrm{~ms}$ & $192 \mathrm{~ms}$ & $455 \mathrm{~ms}$ \\
address & $15 \mathrm{MB}$ & $429 \mathrm{~ms}$ & $68 \mathrm{~ms}$ & $139 \mathrm{~ms}$ \\
SUAS & $13 \mathrm{MB}$ & $232 \mathrm{~ms}$ & $19 \mathrm{~ms}$ & $13 \mathrm{~ms}$ \\
ORTCA & $7.7 \mathrm{MB}$ & $154 \mathrm{~ms}$ & $16 \mathrm{~ms}$ & $6 \mathrm{~ms}$ \\
\hline
\end{tabular}

Fig. 10. Parsing times of XML files (in log scale on the left).

is a start-tag with an attribute with name id and value 1. Below is a snippet of the related XML grammar in ANTLR.

element :

'<' Name attribute* '>' content '<' '/' Name '>' | '<' Name attribute* '/>' ;

To expose the nesting structure within XML, we add an additional step between ANTLR lexing and VPG parsing.

$$
\text { Input file } \stackrel{\text { ANTLR Lexer }}{\longrightarrow} \text { ANTLR tokens } \stackrel{V P G \text { Lexer }}{\longrightarrow} \text { VPG tokens } \stackrel{V P G \text { Parser }}{\longrightarrow} \text { Results. }
$$

The step of VPG lexing coalesces tokens for a single XML tag into a single token. For example, $<p>$ becomes a single token and is marked as a call symbol. For attributes inside tags, they are processed and attached as tags' semantic values for the following parsing step. The following shows a snippet of our adapted XML grammar.

element : <TagOpen content TagClose> | TagSingle ;

The VPG tokens are declared as follows.

TagOpen $=$ '<' Name attribute* ' $>$ ' ;

TagClose $=$ '<' ' $/$ ' Name ' $>$ ' ;

TagSingle $=$ '<' Name attribute* ' $/>$ ' ;

For evaluation, we used the real-world XML files provided by the VTD-XML benchmarks ${ }^{9}$, which consist of a wide selection of 23 files ranging from $1 \mathrm{~K}$ to $73 \mathrm{MB}$. The parsing times are presented in Figure 10; Appendix G of the companion technical report (Jia et al. 2021) shows the results for the full test set. Similar to JSON, VPG parsing on XML files is much faster than ANTLR parsing; on the 5 largest XML files, VPG parsing is about 5 times faster; on smaller files, the gap is even larger.

\subsection{Comparison with ANTLR on Parsing HTML Files}

A snippet of the HTML grammar in ANTLR is listed below:

htmlElement:

'<' TAG_NAME htmlAttribute* ('>' (htmlContent '<' '/' TAG_NAME '>')? | '/' '>' ) ; htmlContent: htmlChardata? ((htmlElement | CDATA | htmlComment) htmlChardata?)* ; Similar to the XML grammar, the HTML grammar allows self-closing tags such as $\langle\mathrm{br} /\rangle$. However, the HTML grammar in addition allows optional end tags, which is not allowed in XML. For example, the HTML tag <input type="submit" value="Ok"> cannot have a matching end tag according

\footnotetext{
${ }^{9}$ https://vtd-xml.sourceforge.io/2.3/benchmark_2.3_parsing_only.html.
} 


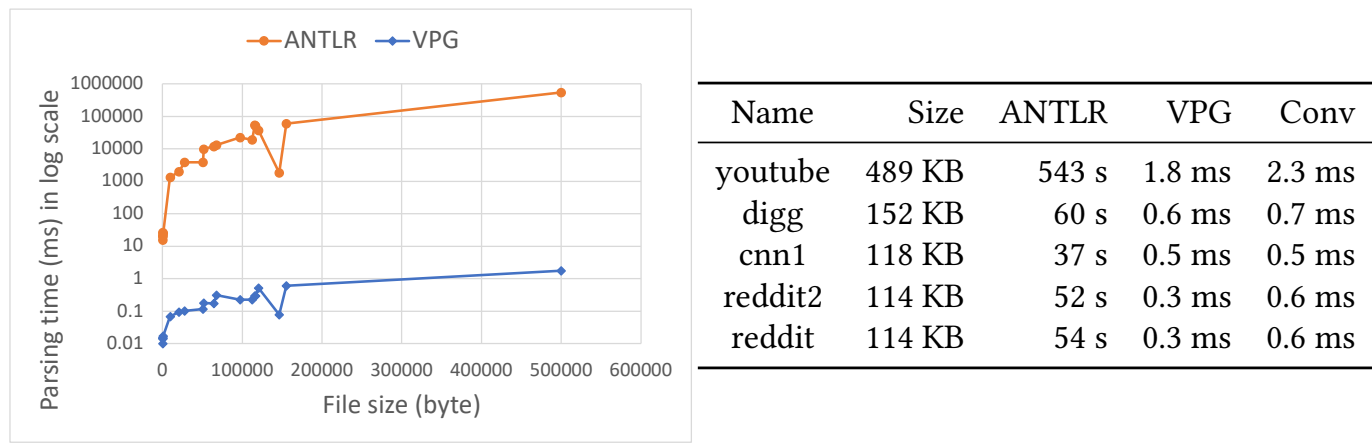

Fig. 11. Parsing times of HTML files (in log scale on the left).

to the HTML standard. Although this kind of tags is also "self-closing", we will use the terminology of optional end tags since that is how the official HTML5 standard describes it. As will be shown in our experimental data, the complexity in this grammar makes ANTLR's parsing of HTML files extremely slow.

Similar to the XML case, we introduced a VPG lexer to coalesce tokens for a single tag into a single token. However, the optional end-tags introduce additional complexity. To explain, let us first examine the relevant part of the VPG grammar:

htmlElement = TagPlain | <TagOpen htmlElement TagClose> | TagSingle ;

The VPG tokens are declared as follows.

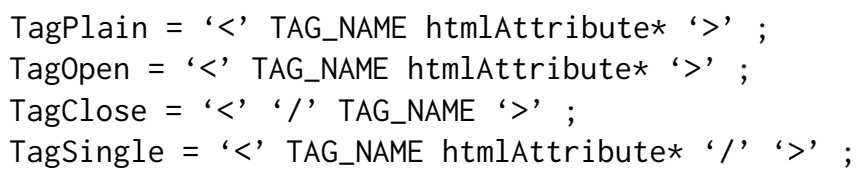

TagPlain is for HTML tags that cannot have matching end-tags, and TagSingle is for self-closing tags. The VPG lexer first merges ANTLR tokens related to a single tag, and then determines which tags are call symbols, return symbols, and plain symbols. A start tag with no matching end tag is marked as a plain symbol in this process. This is implemented with a straightforward method: the first $k$ open HTML tags are matched with the last $k$ close HTML tags, and the rest open HTML tags are viewed as plain symbols, where $k$ is the number of close HTML tags in the file (the number of close tags is always less than or equal to the number of open tags).

For evaluation, we used the 19 real-world HTML files provided in ANTLR's repository ${ }^{10}$. The parsing times are presented in Figure 11. The conversion times of the parse trees are shown in the "Conv" column. As we can see, our VPG parser significantly outperforms the ANTLR parser, with more than 4 orders of magnitude of difference. We emphasize that in our evaluation the VPG parser and the ANTLR parser accept the same HTML files, and they produce the same parse trees with the help of a converter.

From the ANTLR profiling tool, we found that around 99\% time cost by ANTLR is in the prediction of

\section{(htmlContent TAG_OPEN TAG_SLASH TAG_NAME TAG_CLOSE)?}

in the rule of "htmlElement", which triggers many lookahead symbols, and also many DFA cache misses (around a miss rate of 90\%). Appendix $\mathrm{H}$ of the companion technical report (Jia et al. 2021)

\footnotetext{
${ }^{10} \mathrm{https} / /$ github.com/antlr/grammars-v4/tree/master/html/examples
} 
Table 1. Parsing times of 5 largest JSON files. "SpiderM" stands for "SpiderMonkey", and "JSCore" for "JavaScriptCore".

\begin{tabular}{crrrrrrrr}
\hline \multirow{2}{*}{ Name } & Size & $\begin{array}{c}\text { ANTLR } \\
\text { Lex }\end{array}$ & $\begin{array}{r}\text { VPG } \\
\text { Parse }\end{array}$ & Lex+Parse & SpiderM & JSCore & V8 & Chakra \\
\hline JSON.parse & $7.0 \mathrm{MB}$ & $130 \mathrm{~ms}$ & $63 \mathrm{~ms}$ & $193 \mathrm{~ms}$ & $118 \mathrm{~ms}$ & $139 \mathrm{~ms}$ & $76 \mathrm{~ms}$ & $88 \mathrm{~ms}$ \\
airlines & $4.7 \mathrm{MB}$ & $81 \mathrm{~ms}$ & $27 \mathrm{~ms}$ & $108 \mathrm{~ms}$ & $74 \mathrm{~ms}$ & $95 \mathrm{~ms}$ & $42 \mathrm{~ms}$ & $56 \mathrm{~ms}$ \\
educativos & $4.1 \mathrm{MB}$ & $108 \mathrm{~ms}$ & $19 \mathrm{~ms}$ & $128 \mathrm{~ms}$ & $71 \mathrm{~ms}$ & $421 \mathrm{~ms}$ & $45 \mathrm{~ms}$ & $49 \mathrm{~ms}$ \\
canada & $2.1 \mathrm{MB}$ & $45 \mathrm{~ms}$ & $15 \mathrm{~ms}$ & $60 \mathrm{~ms}$ & $57 \mathrm{~ms}$ & $68 \mathrm{~ms}$ & $34 \mathrm{~ms}$ & $44 \mathrm{~ms}$ \\
citm_catalog & $1.6 \mathrm{MB}$ & $39 \mathrm{~ms}$ & $9 \mathrm{~ms}$ & $47 \mathrm{~ms}$ & $34 \mathrm{~ms}$ & $71 \mathrm{~ms}$ & $28 \mathrm{~ms}$ & $25 \mathrm{~ms}$ \\
\hline
\end{tabular}

Table 2. Parsing times of 5 largest XML files. "HP2" stands for "HTMLParser2".

\begin{tabular}{crrrrrrrr}
\hline \multirow{2}{*}{ Name } & Size & $\begin{array}{c}\text { ANTLR } \\
\text { Lex }\end{array}$ & $\begin{array}{r}\text { VPG } \\
\text { Parse }\end{array}$ & Lex+Parse & Fast-XML & Libxmljs & SAX-JS & HP2 \\
\hline po & $73 \mathrm{MB}$ & $1812 \mathrm{~ms}$ & $425 \mathrm{~ms}$ & $2238 \mathrm{~ms}$ & $3278 \mathrm{~ms}$ & $897 \mathrm{~ms}$ & $6618 \mathrm{~ms}$ & $1827 \mathrm{~ms}$ \\
cd & $26 \mathrm{MB}$ & $732 \mathrm{~ms}$ & $192 \mathrm{~ms}$ & $924 \mathrm{~ms}$ & $1298 \mathrm{~ms}$ & $419 \mathrm{~ms}$ & $2103 \mathrm{~ms}$ & $735 \mathrm{~ms}$ \\
address & $15 \mathrm{MB}$ & $367 \mathrm{~ms}$ & $68 \mathrm{~ms}$ & $435 \mathrm{~ms}$ & $584 \mathrm{~ms}$ & $196 \mathrm{~ms}$ & $1012 \mathrm{~ms}$ & $331 \mathrm{~ms}$ \\
SUAS & $13 \mathrm{MB}$ & $237 \mathrm{~ms}$ & $19 \mathrm{~ms}$ & $256 \mathrm{~ms}$ & $254 \mathrm{~ms}$ & $182 \mathrm{~ms}$ & $1214 \mathrm{~ms}$ & $169 \mathrm{~ms}$ \\
ORTCA & $7.7 \mathrm{MB}$ & $142 \mathrm{~ms}$ & $16 \mathrm{~ms}$ & $157 \mathrm{~ms}$ & $138 \mathrm{~ms}$ & $91 \mathrm{~ms}$ & $665 \mathrm{~ms}$ & $89 \mathrm{~ms}$ \\
\hline
\end{tabular}

shows the profiler result for "bbc.com.html". This HTML evaluation shows the power of VPGs in designing practical language parsers, due to their ability of linear-time parsing.

Summary of comparison with ANTLR. Our performance evaluation shows that our VPG parsing library generates parsers that run significantly faster than those generated by ANTLR on grammars that can be converted to VPGs, such as JSON, XML, and HTML.

\subsection{Comparison with Hand-Crafted Parsers}

We also compared VPG parsers with hand-crafted parsers for JSON and XML documents. For JSON, we compared with four mainstream JavaScript engines (V8, Chakra, JavaScriptCore, and SpiderMonkey) and evaluated them on the JSON files in Section 7.1. For XML, we compared with four popular XML parsers (fast-xml-parser, libxmljs, sax-js, and htmlparser2) ${ }^{11}$, and evaluated them with the XML files in Section 7.2.

The evaluation results for the largest files are shown in Table 1 and Table 2; the full results are in Appendix G of the companion technical report (Jia et al. 2021). Note that the hand-crafted parsers can process raw texts directly, while our VPG parsers process the tokens generated by ANTLR's lexers. Therefore, we show separately the lexing time of ANTLR (column "ANTLR Lex"), the parsing time of VPG parsing (column "VPG Parse"), and the combined time (column "Lex+Parse"). From the results, we can see that although the total time of VPG parsing is not the shortest among all parsers, the parsing time alone is. Thus, VPG parsers show promising potential in performance, with additionally verified correctness over hand-crafted parsers. The total parsing time can be reduced by replacing ANTLR's lexer with a faster, customized lexer, since the parsing time of VPG

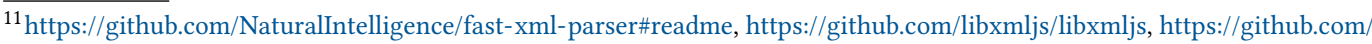
isaacs/sax-js, and https://github.com/fb55/htmlparser2.
} 
is shorter than the lexing time. Also, combining the lexing and parsing steps, as is common in hand-crafted parsers, can usually improve the overall time.

\section{LIMITATIONS AND FUTURE WORK}

As noted earlier, the correctness of our VPG-based parser generator is verified in Coq. Correctness means that if the generated parser constructs a parse tree, it must be a valid parse tree according to the input VPG, and vice versa. However, there are gaps between our VPG parser generator's Coq formalization of and its implementation in OCaml. First, the implementation takes tagged CFGs as input and translates tagged CFGs to VPGs; this translation algorithm has not been formally modeled and verified in Coq. Second, the implementation uses efficient data structures for performance, while their Coq models use equivalent data structures that are slower but easier for reasoning. For example, the OCaml implementation uses hash tables for storing transition tables of the two PDAs to have efficient search (with $O(1)$ search complexity), while the Coq counterpart uses a balanced tree (with $O(\log (n))$ search complexity) provided as a Coq library. These gaps prevent us from directly extracting OCaml code from the Coq formalization.

Our parsing algorithm requires a VPG as the input grammar. Compared to a CFG, a VPG requires partitioning terminals into plain, call, and return symbols. Some CFGs may not admit such kind of partitioning; the same terminal may require different stack actions for different input strings. In particular, all languages recognized by VPGs belong to the set of deterministic context-free languages, which is a strict subset of context-free languages (the classic example that separates CFL from DCFL is $\left.\left\{a^{i} b^{j} c^{k} \mid i \neq j \vee j \vee k\right\}\right)$. We plan to extend our preliminary study on ANTLR grammars to understand how much of the syntax of practical computer languages (e.g., programming languages and file formats) can be described by VPGs.

The parsing in our VPG framework is performed in three steps: construction of a parse forest (with possible invalid edges), pruning of the parse forest, and extraction of parse trees. This design simplifies formal verification but adds a pruning step. We believe the pruning step can be possibly removed by redesigning our parser generator, where the recognizer is extended to a transducer that generates semantic actions and the execution of those semantic actions builds the parse forest directly.

The translation algorithm from tagged CFGs to VPGs is sound but not complete. In general, it is an open problem to determine whether a CFG can be translated to a VPG, and to infer the call and return symbols automatically.

\section{CONCLUSIONS}

In this paper, we present a recognizer and a formally verified parser generator for visibly pushdown grammars. The parsing algorithm is largely enlightened by the recognizer, with several trade-offs to simplify the structure and reduce the burden of formal verification. We also provide a surface grammar called tagged CFGs and a translator from tagged CFGs to VPGs. We show that when a format can be modeled by a VPG and its call and return symbols can be identified, VPG parsing provides competitive performance and sometimes a significant speed-up.

\section{ACKNOWLEDGMENT}

The authors would like to thank anonymous reviewers for their insightful comments. This work was supported by DARPA research grant HR0011-19-C-0073.

\section{REFERENCES}

Rajeev Alur and P. Madhusudan. 2009. Adding Nesting Structure to Words. J. ACM 56, 3, Article 16 (May 2009), 43 pages. https://doi.org/10.1145/1516512.1516518 
Janusz A. Brzozowski. 1964. Derivatives of Regular Expressions. J. ACM 11, 4 (Oct. 1964), 481-494. https://doi.org/10.1145/ 321239.321249

John Cocke. 1969. Programming Languages and Their Compilers: Preliminary Notes. New York University, USA.

F. L. Deremer. 1969. PRACTICAL TRANSLATORS FOR LR(K) LANGUAGES. Technical Report. USA.

Jay Earley. 1970. An Efficient Context-Free Parsing Algorithm. Commun. ACM 13, 2 (Feb. 1970), 94-102. https://doi.org/10. $1145 / 362007.362035$

Romain Edelmann, Jad Hamza, and Viktor Kunčak. 2020. Zippy LL(1) Parsing with Derivatives. In Proceedings of the 41st ACM SIGPLAN Conference on Programming Language Design and Implementation (London, UK) (PLDI 2020). Association for Computing Machinery, New York, NY, USA, 1036-1051. https://doi.org/10.1145/3385412.3385992

Bryan Ford. 2004. Parsing Expression Grammars: A Recognition-Based Syntactic Foundation. SIGPLAN Not. 39,1 (Jan. 2004), 111-122. https://doi.org/10.1145/982962.964011

Ian Henriksen, Gianfranco Bilardi, and Keshav Pingali. 2019. Derivative Grammars: A Symbolic Approach to Parsing with Derivatives. Proc. ACM Program. Lang. 3, OOPSLA, Article 127 (Oct. 2019), 28 pages. https://doi.org/10.1145/3360553

Xiaodong Jia, Ashish Kuma, and Gang Tan. 2021. A Derivative-based Parser Generator for Visibly Pushdown Grammars. arXiv:2109.04258 [cs.PL]

Jacques-Henri Jourdan, François Pottier, and Xavier Leroy. 2012. Validating LR (1) parsers. 397-416. https://doi.org/10. 1007/978-3-642-28869-2_20

Tadao Kasami. 1965. An Efficient Recognition and Syntax-Analysis Algorithm for Context-Free Languages. Technical Report. Air Force Cambridge Research Laboratory.

Adam Koprowski and Henri Binsztok. 2011. TRX: A Formally Verified Parser Interpreter. Logical Methods in Computer Science 7 (05 2011). https://doi.org/10.2168/LMCS-7(2:18)2011

Sam Lasser, Chris Casinghino, Kathleen Fisher, and Cody Roux. 2019. A Verified LL(1) Parser Generator. In 10th International Conference on Interactive Theorem Proving (ITP 2019) (Leibniz International Proceedings in Informatics (LIPIcs), Vol. 141), John Harrison, John O'Leary, and Andrew Tolmach (Eds.). Schloss Dagstuhl-Leibniz-Zentrum fuer Informatik, Dagstuhl, Germany, 24:1-24:18. https://doi.org/10.4230/LIPIcs.ITP.2019.24

Sam Lasser, Chris Casinghino, Kathleen Fisher, and Cody Roux. 2021. CoStar: A Verified ALL $\left(^{*}\right)$ Parser. In Proceedings of the 42nd ACM SIGPLANInternational Conference on Programming Language Design and Implementation (Virtual, Canada) (PLDI 2021). Association for Computing Machinery, New York, NY, USA, 420-434. https://doi.org/10.1145/3453483.3454053

Matthew Might, David Darais, and Daniel Spiewak. 2011. Parsing with Derivatives: A Functional Pearl. In Proceedings of the 16th ACM SIGPLAN International Conference on Functional Programming (Tokyo, Japan) (ICFP '11). Association for Computing Machinery, New York, NY, USA, 189-195. https://doi.org/10.1145/2034773.2034801

Greg Morrisett, Gang Tan, Joseph Tassarotti, Jean-Baptiste Tristan, and Edward Gan. 2012. RockSalt: Better, Faster, Stronger SFI for the X86. SIGPLAN Not. 47, 6 (June 2012), 395-404. https://doi.org/10.1145/2345156.2254111

Scott Owens, John Reppy, and Aaron Turon. 2009. Regular-Expression Derivatives Re-Examined. F. Funct. Program. 19, 2 (March 2009), 173-190. https://doi.org/10.1017/S0956796808007090

Terence Parr, Sam Harwell, and Kathleen Fisher. 2014. Adaptive LL $\left(^{*}\right)$ Parsing: The Power of Dynamic Analysis. SIGPLAN Not. 49, 10 (Oct. 2014), 579-598. https://doi.org/10.1145/2714064.2660202

Theofilos Petsios, Adrian Tang, Salvatore Stolfo, Angelos D. Keromytis, and Suman Jana. 2017. NEZHA: Efficient DomainIndependent Differential Testing. In 2017 IEEE Symposium on Security and Privacy (SP). 615-632. https://doi.org/10.1109/ SP.2017.27

Tahina Ramananandro, Antoine Delignat-Lavaud, Cedric Fournet, Nikhil Swamy, Tej Chajed, Nadim Kobeissi, and Jonathan Protzenko. 2019. EverParse: Verified Secure Zero-Copy Parsers for Authenticated Message Formats. In 28th USENIX Security Symposium (USENIX Security 19). USENIX Association, Santa Clara, CA, 1465-1482. https://www.usenix.org/ conference/usenixsecurity19/presentation/delignat-lavaud

Daniel H. Younger. 1967. Recognition and parsing of context-free languages in time n3. Information and Control 10, 2 (1967), 189-208. https://doi.org/10.1016/S0019-9958(67)80007-X 